\title{
REAL OPERATOR ALGEBRAS AND REAL POSITIVE MAPS
}

\author{
DAVID P. BLECHER AND WORAWIT TEPSAN
}

\begin{abstract}
We present some foundations for a theory of real operator algebras and real Jordan operator algebras, and the various morphisms between these. A common theme is the ingredient of real positivity from papers of the first author with Read, Neal, Wang, and other coauthors, which we import to the real scalar setting here.
\end{abstract}

\section{INTRODUCTION}

In a 2016 paper [39, Rosenberg commented "Over the last thirty years ... it has become apparent that real $C^{*}$-algebras have a lot of extra structure not evident from their complexifications. At the same time, interest in real $C^{*}$-algebras has been driven by a number of compelling applications." He goes on to mention the classification of manifolds of positive scalar curvature, representation theory, the study of orientifold string theories, and the real Baum-Connes conjecture. Even the problem of whether a complex $C^{*}$-algebra is the complexification of a real $C^{*}$ algebra is a difficult one, with contributions by Connes [21, Jones, Størmer, etc.

On the other hand, Böttcher and Pietsch comment in [11] that the impression sometimes given that results about complex Hilbert spaces carry over mutatis mutandis to the real case, should not be taken too literally. They go on to point out that searching in Mathematical Reviews for publications whose title contains the term 'Hilbert space' yields an output of approximately 10000, while asking for 'real Hilbert space' reduces the result to 100 (we have changed their numbers to the current ones). They suggest that this indicator, while very rough, nonetheless displays the shameful treatment of the real case. Similarly there has been very little work done on real operator algebras relatively speaking, and there is a frequent lack of appreciation of the considerable difficulties that can arise.

In the present paper we present some foundations for a theory of real operator algebras and real Jordan operator algebras, and the various morphisms between these (see also [42, 44]). A common theme is the ingredient of real positivity from papers of the first author with Read in [12, 13, 14, (see also e.g. [4, 5, 8, 16, 9, 10, 6]) in the complex case, which we import to the real scalar setting here. A bounded linear operator on a Hilbert space $H$ is real positive if $T+T^{*} \geq 0$. Real positivity is intended to be, for nonselfadjoint operator algebras or unital operator spaces, a substitute for positivity in $C^{*}$-algebras. One indication that real positivity will be useful in the real theory is the fact (which follows from e.g. Lemma 5.14), that

2020 Mathematics Subject Classification. Primary 17C65 , 46L05, 46L70, 47L05, 47L07, 47L30, 47L70; Secondary: 46H10, 46B40, 46L07, 46L30, 47B92, 47L75.

Key words and phrases. Operator algebra, real Jordan operator algebra, real operator system, real completely bounded maps, real positive maps, approximate identities.

Supported by a Simons Foundation Collaboration Grant. 
states on a real $C^{*}$-algebra are the norm 1 real positive functionals, but they are not the norm 1 positive functionals (see the example above Proposition 4.1 in [40]).

An associative real (resp. complex) operator algebra is a possibly nonselfadjoint closed real (resp. complex) subalgebra of $B(H)$, for a real (resp. complex) Hilbert space $H$. See e.g. [7] for the complex case of this theory, and 42 for a preliminary study of the real case. By a real (resp. complex) Jordan operator algebra we mean a norm-closed real (resp. complex) Jordan subalgebra $A$ of a real (resp. complex) $C^{*}$-algebra, namely a norm-closed real (resp. complex) subspace closed under the 'Jordan product' $a \circ b=\frac{1}{2}(a b+b a)$. Or equivalently, with $a^{2} \in A$ for all $a \in A$ (this follows since $\left.a \circ b=\frac{1}{2}\left((a+b)^{2}-a^{2}-b^{2}\right)\right)$. A characterization of these algebras is given in [44, Section 4.3] and in Theorem 2.1 in [9, 16. The selfadjoint case, that is, closed selfadjoint real (resp. complex) subspaces of a real (resp. complex) $C^{*}$-algebra which are closed under squares, will be called real (resp. complex) $J C^{*}$ algebras. Complex $J C^{*}$-algebras have a large literature, see e.g. 19, 26, 45, for references. The theory of (possibly nonselfadjoint) Jordan operator algebras over the complex field was initiated in [16, 9] (see also [17, 46, for some additional results, complements, etc).

Of course every operator algebra is a Jordan operator algebra. Jordan operator algebras are the most general setting for many of the results below. Thus we state such results in this setting; the reader who does not care about nonassociative algebras should simply restrict to the associative case. Indeed the statement of some of our results contain the phrase '(Jordan) operator algebra', this invites the reader to simply ignore the word 'Jordan'. Another reason to consider real Jordan operator algebras is that there are many examples, for example in quantum physics. In addition to the complex examples there are the real JC-algebras (cf. [26, 45]), namely Jordan algebras of selfadjoint operators on a real Hilbert space. We mention for example spin systems: families of selfadjoint unitaries $\left\{u_{i}: i \in I\right\}$ on a real Hilbert space $H$ with $u_{i} \circ u_{j}=0$ if $i \neq j$. For example the Pauli matrices, and the usual appropriate tensor product of these, are good examples of these. The closed real span of such a family, and the identity $I$, is a real unital Jordan operator subalgebra of $B(H)$, which is isomorphic to a Hilbert space (see e.g. p. 175 in [37]).

We are able to include a rather large number of results in a relatively short manuscript since many proofs are similar to their complex counterparts, and thus we often need only discuss the new points that arise. Note that real (Jordan) operator algebras can be treated to some extent in the framework of complex (Jordan) operator algebras with an involution [18. Indeed the study of these real algebras is 'the same as' (in some sense-but do not take this too literally) the study of complex (Jordan) operator algebras with a period 2 conjugate linear (Jordan) automorphism, which is isometric or completely isometric. Indeed the fixed points of such an automorphism is a real (Jordan) operator algebra, and conversely the complexification of a real (Jordan) operator algebra has such an automorphism $x+i y \mapsto x-i y$. Similarly for dual real (Jordan) operator algebras, except now the automorphism is weak* continuous.

Thus often a result about real spaces is proved by applying the complex variant of the result to the complexification. We will rarely spend much time on such results, focusing rather on situations where complexification fails. In the latter case one may try to copy the proof of the complex variant, or invent a completely new argument, or attempt a mixture of these two. Indeed there are a few pitfalls that one must 
beware of, and standard tricks in the complex case that fail for real spaces. For example, for an operator $T$ on a real Hilbert space $H$ the condition $\langle T \xi, \xi\rangle \geq 0$ for every $\xi \in H$ does not imply that $T \geq 0$. Indeed arguments involving states or numerical range often do not work in the real case. E.g. the matrix $E_{12}-E_{21}$ takes value 0 at every real state on $M_{2}(\mathbb{R})$, but it is not Hermitian (selfadjoint). We know of no simple test for $T \in B(H)$ to be positive or selfadjoint in the real case. Thus some aspects and directions in the theory do not generalize very well to the real case, as we shall see e.g. in parts of Section 5 .

We do not attempt to be exhaustive, rather we are content to prove enough results here so that the reader who is familiar with the complex theory from the literature listed in the third paragraph will feel, after finishing our paper, that they have a good grasp of how the real case works out.

In Section 2 we give some basic results on real completely positive maps on real operator algebras and real Jordan operator algebras, and completely positive maps on real operator systems. In Section 3 we establish the variant of Meyer's unitization theorem for contractive homomorphisms on a real Jordan operator algebra. Namely for a real Jordan subalgebra $A$ of $B(H)$ not containing $I_{H}$, any contractive Jordan homomorphism from $A$ to $B(K)$ extends to a unital contractive homomorphism from $A+\mathbb{R} I_{H}$ to $B(K)$. This result does not just follow by complexificationwe have to exploit the Cayley transform as in Meyer's remarkable original proof. This implies in particular that the unitization of a real Jordan operator algebra is uniquely defined up to isometric algebra isomorphism. In Section 4 we study contractive approximate identities (or cais), generalizing to the real case some of the main results from [16] (and from the sequence of papers mentioned above) about cais and about approximately unital algebras.

In Section 5 we study real positivity and real positive maps, generalizing to the real case some of the main results from [10, Section 2]. Thus Section 5 is the appropriate variant for real Jordan operator algebras and real unital operator spaces, of the theory of positive (but not completely positive maps) on complex $C^{*}$-algebras or operator systems. It turns out that if we are interested in real positive maps as opposed to RCP maps (see Section 2) then an extra condition is usually needed for the real theory, namely the notion of systematic real positive. However even then some obstacles emerge that do not exist in the complex case. We give several examples of bad behavior. For example unital contractions on a real operator system need not be selfadjoint, nor positive, and need not extend to a contraction on the unitization. Also real positive maps (resp. positive selfadjoint maps) need not extend to a real positive map (resp. positive selfadjoint map) on a complexification. We also list some questions that we do not know the answer to but probably are also evidence of malaise in the real case at this level of generality.

In the remaining part of Section 1 we give some background and notation. The underlying scalar field is usually $\mathbb{R}$, and all spaces, maps or operators in this paper are usually $\mathbb{R}$-linear. For background on completely bounded maps, complex operator spaces, and associative operator algebras, we refer the reader to [7, 22, 35, 37]. For complex $C^{*}$-algebras the reader could consult e.g. 36. For background on 'selfadjoint' nonassociative and 'Jordan type' algebras see e.g. [19] and references therein. For the theory of possibly nonselfadjoint complex Jordan operator algebras the reader will also want to consult [16] frequently for background, notation, etc, and will often be referred there for various results that are used here. 
The letters $H, K$ are reserved for real Hilbert spaces. A projection in an algebra is always an orthogonal projection (so $p=p^{2}=p^{*}$ ). A (possibly nonassociative) normed algebra $A$ is unital if it has an identity 1 of norm 1, and a map $T$ is unital if $T(1)=1$. We write $X_{+}$for the positive operators (in the usual sense) that happen to belong to $X$. A Jordan homomorphism $T: A \rightarrow B$ between Jordan algebras is a linear map satisfying $T(a b+b a)=T(a) T(b)+T(b) T(a)$ for $a, b \in A$, or equivalently, that $T\left(a^{2}\right)=T(a)^{2}$ for all $a \in A$ (the equivalence follows by applying $T$ to $(a+b)^{2}$ ). We write $M_{n}(X)$ for the space of $n \times n$ matrices over a space $X$.

Some general background on differences between 'real and complex functional analysis' may be found in the recent survey 33 that was not available at the time of submission of our paper. We will assume that the reader is familiar with some basics from the theory of real $C^{*}$-algebras 31, in particular that a real $C^{*}$-algebra $B$ has a 'unique $C^{*}$-algebra complexification' such that the map $x+i y \mapsto x-i y$ is isometric (here $x, y \in B$ ). This unique complexification may be equivalently characterized in terms of period 2 conjugate linear $*$-automorphisms on complex $C^{*}$-algebras. We will often use facts from that theory that will be evident for readers for the complex case, and whose real versions may all be found in [31. If $X$ is a subspace of a (real or complex) $C^{*}$-algebra $B$ then we write $C^{*}(X)$ or $C_{B}^{*}(X)$ for the $C^{*}$-subalgebra of $B$ generated by $X$.

Although we shall not use this we mention as a background fact that the first author together with Mehrdad Kalantar were able to improve on the characterization of commutative real $W^{*}$-algebras from [31] (see e.g. Theorem 6.3.1 there, and e.g. 29, 2]). This may be new in this full generality (e.g. [31, 2] seem to consider separably acting algebras). Indeed the commutative real $W^{*}$-algebras come from the commutative complex $W^{*}$-algebras regarded as real algebras:

Theorem 1.1. Any commutative real $W^{*}$-algebra is (real) *-isomorphic to the direct sum of a commutative complex $W^{*}$-algebra, and the set of selfadjoint elements in a commutative complex $W^{*}$-algebra. This may be written as $L^{\infty}(X, \mu, \mathbb{C}) \oplus$ $L^{\infty}(Y, \nu, \mathbb{R})$. Here $(X, \mu)$ and $(Y, \mu)$ are localizable (or Radon) measure spaces (and we allow one of these to be the empty set, and in this case interpret the $L^{\infty}$-space to be (0)).

Ruan initiated the study of real operator spaces in 40, 41, and this study was continued in 42. A real operator space may either be viewed as a real subspace of $B(H)$, or abstractly as a vector space with a norm $\|\cdot\|_{n}$ on $M_{n}(X)$ for each $n \in \mathbb{N}$ (satisfying Ruan's axioms [40]). Sometimes the sequence of norms $\left(\|\cdot\|_{n}\right)$ is called the operator space structure. All spaces in the present paper are such operator spaces, although sometimes we will not care about the higher matrix norms. We will say that an operator space complexification $X_{c}=X+i X$ of an operator space $X$ is completely reasonable if the map $\iota_{X}: x+i y \mapsto x-i y$ is a 'complete isometry', for $x, y \in X$. Ruan proved that a real operator space $X$ possesses a completely reasonable operator space complexification, which is unique up to complete isometry. This unique complexification may be identified up to real complete isometry with the operator subspace of $M_{2}(X)$ of matrices of form

$$
\left[\begin{array}{cc}
x & -y \\
y & x
\end{array}\right]
$$

for $x, y \in X$. 
One may define an operator algebra complexification (resp. Jordan operator algebra complexification) of a real operator algebra (resp. Jordan operator algebra) $A$ to be the complex linear span $A+i A$ of $A$ in $B_{c}$, if $A$ is a subalgebra (resp. Jordan subalgebra) of a real $C^{*}$-algebra $B$. Here $B_{c}$ is the unique $C^{*}$-algebra complexification a couple of paragraphs above. In particular if $A$ is a real subalgebra (resp. Jordan subalgebra) of $B(H)$ for a real Hilbert space $H$ then the operator algebra (resp. Jordan operator algebra) complexification $A+i A$ is a complex subalgebra (resp. Jordan subalgebra) of $B(H)_{c}=B\left(H_{c}\right)$. These definitions have the drawback of being too 'concrete', but are easily made 'more suitable for abstract use' by adding words like 'is isometrically isomorphic (resp. Jordan isomorphic) to' in a couple of places above. One uses 'completely isometrically' here in place of 'isometrically' if one needs to work in the matrix normed (operator space) setting. As mentioned much earlier in the introduction, this complexification can be equivalently defined abstractly in terms of a period 2 conjugate linear isometric (or completely isometric, if one needs to work in the matrix normed setting) automorphism $\theta$ on a complex operator algebra $B$. Here $B$ is spanned by the subspace of fixed points of $\theta$, which we are assuming to be an isometric (or completely isometric) 'copy' of the operator algebra (resp. Jordan operator algebra) $A$. We say that such $B$ is an operator algebra (resp. Jordan operator algebra) complexification of $A$. We show that then $B$ is (isometrically isomorphic to) a concrete complexification of an isometric copy of $A$ of the type described at the start of this paragraph. Suppose that $B$ acts on a Hilbert space $H$, and set $D=\{(b, \overline{\theta(b)}) \in B(H) \oplus \overline{B(H)}: b \in B\}$, a copy of $B$. One may use the fact that $\overline{B(H)}=B(\bar{H}) *$-isomorphically, where $\overline{B(H)}$ is the $C^{*}$-algebra in e.g. the remark after Proposition 2.1 in [18. Define $C$ to be the $C^{*}$-algebra generated by $D$, and let $\rho$ be the period 2 conjugate linear *automorphism on $C$ which is the restriction of the *-automorphism $(S, \bar{T}) \mapsto(T, \bar{S})$ on $B(H) \oplus \overline{B(H)}$. The fixed points of $\rho$ are a real $C^{*}$-algebra $M$ with complexification $C$, and $A$ may be viewed as its copy $\{(a, \overline{\theta(a)}): a \in A\}$ in $M$. Moreover $\rho$ extends the copy of $\theta$ on $D$. Thus the complexification of this copy of $A$ as described at the start of this paragraph, is isometrically isomorphic to $B$. The same proof works in the completely isometric case.

It is important to realize that the operator algebra (resp. Jordan operator algebra) complexification $A_{c}$ is not uniquely defined up to isometric isomorphism or isometric Jordan isomorphism. That is, the complexification of a real operator algebra $A$ is not uniquely defined at the Banach algebra level. This is clear for example from Proposition 2.10 If however we also take the operator space structure of $A$ into account then $A_{c}$ is uniquely defined up to completely isometric algebra (resp. Jordan algebra) isomorphism. This follows for example by [41, Theorem 2.1]. If $A \subset B(H)$ is a real Jordan operator algebra then $A_{c}$ may be identified up to real complete isometric isomorphism with the Jordan subalgebra of matrices of the form (1.1) in $M_{2}(A) \cap M_{2}(B(H))$. Note that $A \cap\left(A_{c}\right)^{-1}=A^{-1}$, since e.g. if $a(b+i c)=1$ then $i a c=0$ and $a b=1$.

If $A$ is a Jordan operator subalgebra of $B(H)$, then the diagonal $\Delta(A)=A \cap A^{*}$ is a $J C^{*}$-algebra. An element $q$ in a Jordan operator algebra $A$ is a projection if $q^{2}=q$ and $\|q\|=1$ (so these are just the orthogonal projections on the Hilbert space which $A$ acts on, and which are in $A$ ). Clearly $q \in \Delta(A)$. A Jordan contractive approximate identity (or $J$-cai for short) for a Jordan operator algebra $A$, is a net $\left(e_{t}\right)$ of contractions (i.e. elements of norm $\leq 1$ ) with $e_{t} \circ a \rightarrow a$ for all $a \in A$. If 
a J-cai for $A$ exists then $A$ is called approximately unital. See Lemma 4.2 for the main result on such approximate identities. One consequence of this result is that an associative operator algebra has a cai if and only if it has a J-cai.

We say that an operator $x$ in $B(H)$ is real positive if $x+x^{*} \geq 0$. (Sometimes this is called being accretive.) This is the same as $x$ being real positive in $B\left(H_{c}\right)$, and hence it follows that the characterizations mentioned in [5, Lemma 2.4] of real positive elements in $B(H)$ are still valid. For example, $x+x^{*} \geq 0$ if and only $\|I-t x\| \leq 1+t^{2}\|x\|^{2}$ for all $t>0$. If $A$ is a unital subspace or (Jordan) subalgebra of $B(H)$ then we define the real positive elements in $A$ to be $\mathfrak{r}_{A}=\left\{x \in A: x+x^{*} \geq 0\right\}$. If $A$ is unital then it follows that the characterizations in [5, Lemma 2.4] are true for $A$, and that the definition of 'real positive' or $\mathfrak{r}_{A}$ does not depend on the particular $B(H)$ that $A$ sits in (isometrically and unitally).

For a (Jordan) operator algebra or unital operator space $A$, because of the uniqueness of unitization up to isometric isomorphism (see Section 3), we can define unambiguously $\mathfrak{F}_{A}=\{a \in A:\|1-a\| \leq 1\}$. Then

$$
\frac{1}{2} \mathfrak{F}_{A}=\{a \in A:\|1-2 a\| \leq 1\} \subset \operatorname{Ball}(A) .
$$

Note that $x \in \mathfrak{F}_{A}$ if and only if $x^{*} x \leq x+x^{*}$. This is because $\|1-a\| \leq 1$ if and only if $(1-a)^{*}(1-a) \leq 1$. It follows that $\mathfrak{F}_{A} \subset \mathfrak{r}_{A}$.

If $T: X \rightarrow Y$ we write $T_{n}$ for the canonical 'entrywise' amplification taking $M_{n}(X)$ to $M_{n}(Y)$. The completely bounded norm is $\|T\|_{\mathrm{cb}}=\sup _{n}\left\|T_{n}\right\|$, and $T$ is contractive (resp. completely contractive) if $\|T\| \leq 1$ (resp. $\|T\|_{\mathrm{cb}} \leq 1$ ). A map $T$ is said to be real positive if it takes real positive elements to real positive elements. We say that it is real completely positive or $R C P$ if $T_{n}$ is real positive for all $n \in \mathbb{N}$. We remark that one may study some aspects of real positivity or real positive maps within the context of the theory of numerical range and generalized numerical range (see e.g. [19, Sections 2.1 and 2.9], or [38, Section 4.3] for some recent results that may be useful in future studies).

If $A$ is a real Jordan subalgebra of a real $C^{*}$-algebra $B$ then $A^{* *}$ with its Arens product is a weak* closed real Jordan subalgebra of the real von Neumann algebra $B^{* *}$. This follows by routine techniques as in the complex case (see 44, Section 4.2] and [16, Section 1]). Since the diagonal $\Delta\left(A^{* *}\right)$ is a real $\mathrm{JW}^{*}$-algebra (that is a weak* closed real $J C^{*}$-algebra), it follows that $A^{* *}$ is closed under meets and joins of projections.

States on a unital real Jordan operator algebra $A$ (that is, unital contractive functionals) extend to states on any Jordan operator algebra complexification $A_{c}$ by the real Hahn-Banach theorem. We will discuss states on approximately unital algebras in Section 4

\section{REAL COMPLETELY POSITIVE MAPS ON REAL OPERATOR ALGEBRAS}

Lemma 2.1. Let $A$ be a real $C^{*}$-algebra and let $A_{c}$ be its complexification. If $x, y \in A$ then $x+i y \geq 0$ in $A_{c}$ if and only if $\left[\begin{array}{cc}x & -y \\ y & x\end{array}\right]$ is positive in $M_{2}(A)$.

Proof. For any complex $C^{*}$-algebra $B$ the map taking the $C^{*}$-subalgebra of $M_{2}(B)$ consisting of $2 \times 2$ matrices of the above form, for $a, b \in B$, to $(a+i b, a-i b)$, is a faithful $*$-homomorphism onto $B \oplus B$. Now set $B=A_{c}$. The matrix in the lemma is positive in $M_{2}(A)$ if and only if it is positive in $M_{2}(A)_{c}=M_{2}\left(A_{c}\right)$, hence if and 
only if $(x+i y, x-i y)$ is positive in $B \oplus B$. Also the map $x+i y \mapsto x-i y$ is a *-automorphism of $B=A_{c}$, hence is positive. Thus the matrix in the lemma is positive if and only if both $x \pm i y \geq 0$, and if and only if $x+i y \geq 0$.

Another proof of the last result may be found in [44, Lemma 3.3.1]. This uses the spectrum and the fact, which is easy to see, that if $x, y \in A$ then $x+i y$ is selfadjoint in $A_{c}$ if and only if $x$ is selfadjoint and $y$ is antisymmetric (i.e. $y^{*}=-y$ ).

Let $X \subset B(H)$ be an operator space which is selfadjoint (that is $x^{*} \in X$ if $x \in X)$. Write $X_{\text {sa }}$ (resp. $X_{\text {as }}$ ) for the selfadjoint (resp. antisymmetric) elements in $X$. Note that $X=X_{\mathrm{sa}} \oplus X_{\text {as }}$ (using the relation $x=\frac{1}{2}\left(x+x^{*}\right)+\frac{1}{2}\left(x-x^{*}\right)$ ).

Lemma 2.2. If $X, Y$ are real selfadjoint operator spaces and $T: X \rightarrow Y$ is real linear then $T$ is selfadjoint if and only if $T\left(X_{\mathrm{sa}}\right) \subset Y_{\mathrm{sa}}$ and $T\left(X_{\mathrm{as}}\right) \subset Y_{\mathrm{as}}$.

Proof. The one direction is obvious. If $T\left(X_{\mathrm{sa}}\right) \subset Y_{\mathrm{sa}}$ and $T\left(X_{\mathrm{as}}\right) \subset Y_{\text {as }}$ and $x=$ $\frac{1}{2}\left(x+x^{*}\right)+\frac{1}{2}\left(x-x^{*}\right)$, then $T\left(\frac{1}{2}\left(x+x^{*}\right)\right) \in Y_{\text {sa }}$ and $T\left(\frac{1}{2}\left(x-x^{*}\right)\right) \in Y_{\text {as }}$. Thus

$$
T(x)^{*}=T\left(\frac{1}{2}\left(x+x^{*}\right)\right)-T\left(\frac{1}{2}\left(x-x^{*}\right)\right)=T\left(x^{*}\right)
$$

as desired.

A real operator system is a selfadjoint unital real subspace $X$ of $B(H)$ for a real Hilbert space $H$ (or of a real unital $C^{*}$-algebra). We say that $x \in X$ is positive if and only if $x$ is positive in $B(H)$; so $X_{+}=X \cap B(H)_{+}$. One usually considers operator systems together with their matrix structure, with morphisms the completely positive selfadjoint maps, or sometimes the completely positive unital selfadjoint maps. The matrix structure consists usually of the positive cones $M_{n}(X)_{+} \subset M_{n}(B(H))_{+}=B\left(H^{(n)}\right)_{+}$, for all $n \in \mathbb{N}$. A real unital operator space is a unital subspace $X$ of a real unital $C^{*}$-algebra (or of $B(H)$ ). Again one usually considers these together with their operator space (i.e. matrix norm) structure, with morphisms the unital completely contractive maps.

A unital selfadjoint map defined on a real operator system $X$ is completely contractive if and only if it is completely positive, by [40, Proposition 4.1]. From this it is easily seen that the positive cone $X_{+}$(and $M_{n}(X)_{+}$for all $n \in \mathbb{N}$ ) is independent of a choice of representation.

If $T: X \rightarrow Y$ then $T_{c}: X_{c} \rightarrow Y_{c}$ is defined as $T_{c}(x+i y)=T(x)+i T(y)$ for $x, y \in A$.

Lemma 2.3. Let $X$ be a real operator system and $T: X \rightarrow B(H)$ be a completely positive map. Then $T_{c}: X_{c} \rightarrow B(H)_{c}$ is completely positive. Also $T$ and $T_{c}$ are selfadjoint and $\|T\|=\|T\|_{\mathrm{cb}}=\|T(1)\|$.

Proof. We will temporarily write $\iota$ for $i \in \mathbb{C}$, to avoid confusion with usual matrix subscripting. Let $w=\left[x_{i j}+\iota y_{i j}\right] \geq 0$ in $M_{n}\left(X_{c}\right)=M_{n}(X)_{c}$, and set $z=\left[\begin{array}{cc}x_{i j} & -y_{i j} \\ y_{i j} & x_{i j}\end{array}\right]$. Then by the Lemma above, $z \geq 0$ in $M_{2 n}(X)$. Since $T$ is completely positive, then

$$
T_{2 n}(z)=\left[\begin{array}{cc}
T\left(x_{i j}\right) & -T\left(y_{i j}\right) \\
T\left(y_{i j}\right) & T\left(x_{i j}\right)
\end{array}\right] \geq 0
$$

in $M_{2 n}(X)$. Thus by the same lemma above, $\left(T_{c}\right)_{n}\left(\left[x_{i j}+\iota y_{i j}\right]\right)=\left[\left[T\left(x_{i j}\right)+\right.\right.$ $\left.\iota T\left(y_{i j}\right)\right] \geq 0$. Thus $T_{c}$ is completely positive. Since a positive map between complex 
operator system is selfadjoint, $T_{c}$ is selfadjoint. Thus $T$ is also selfadjoint. The norm and completely bounded norm of $T_{c}$ equal $\|T(1)\|$ by e.g. [35, Proposition 3.6], hence $\|T\|=\|T\|_{\mathrm{cb}}=\|T(1)\|$.

Proposition 2.4. Let $X$ be a real unital operator space or approximately unital real Jordan operator algebra and $T: X \rightarrow B(H)$ real completely positive. Then $T_{c}$ is real completely positive. Also $T$ has a well defined extension $\tilde{T}$ to $X+X^{*}$ which is selfadjoint and completely positive. Also $\|T\|=\|T\|_{\mathrm{cb}}=\|\tilde{T}\|_{\mathrm{cb}}$. This equals $\|T(1)\|$ in the unital case, and in the nonunital case is $\sup _{t}\left\|T\left(e_{t}\right)\right\|\left(=\lim _{t}\left\|T\left(e_{t}\right)\right\|\right)$ for any $J$-cai $\left(e_{t}\right)$ for $A$.

Proof. We follow the idea and notation of Lemma 2.3 Let $w=\left[x_{i j}+\iota y_{i j}\right]$. If $w+w^{*} \geq 0$ then

$$
\left[\begin{array}{cc}
x_{i j} & -y_{i j} \\
y_{i j} & x_{i j}
\end{array}\right]+\left[\begin{array}{cc}
x_{j i}^{*} & y_{j i}^{*} \\
-y_{j i}^{*} & x_{j i}^{*}
\end{array}\right]=\left[\begin{array}{cc}
x_{i j} & -y_{i j} \\
y_{i j} & x_{i j}
\end{array}\right]+\left[\begin{array}{cc}
x_{i j} & -y_{i j} \\
y_{i j} & x_{i j}
\end{array}\right]^{*} \geq 0
$$

in $M_{2 n}(X)$. Since $T$ is completely real positive,

$$
\left[\begin{array}{cc}
T\left(x_{i j}\right) & -T\left(y_{i j}\right) \\
T\left(y_{i j}\right) & T\left(x_{i j}\right)
\end{array}\right]+\left[\begin{array}{cc}
T\left(x_{i j}\right) & -T\left(y_{i j}\right) \\
T\left(y_{i j}\right) & T\left(x_{i j}\right)
\end{array}\right]^{*} \geq 0 .
$$

Reversing the steps at the start of the proof, but applied to the last matrix, we see that $\left[T\left(x_{i j}\right)+\iota T\left(y_{i j}\right)\right]+\left[T\left(x_{i j}\right)+\iota T\left(y_{i j}\right)\right]^{*} \geq 0$. Hence $T_{c}$ is completely real positive.

In the unital case, by the complex theory (see e.g. [4, Theorem 2.5]) $T_{c}$ is bounded and extends to a selfadjoint completely positive map $X_{c}+\left(X_{c}\right)^{*} \rightarrow B\left(H_{c}\right)$. This restricts to a selfadjoint completely positive map $X+X^{*} \rightarrow B(H)$. By Lemma 2.3 we have $\|\tilde{T}\|_{\mathrm{cb}}=\|T(1)\|=\|\tilde{T}\|$, which implies that the norm and completely bounded norm of $T$ equals $\|T(1)\|$ too.

In the approximately unital real Jordan operator algebra case, by the complex theory (see [10, Lemma 2.1] and [16, Proposition 4.9]) $T_{c}$ is bounded, so $T$ is bounded, and $\left\|T_{c}\right\|=\|T\|=\left\|T_{c}^{* *}(1)\right\|=\left\|T^{* *}(1)\right\|$. Alternatively, let $\widehat{T}_{c}: X_{c}^{* *} \rightarrow$ $B\left(H_{c}\right)$ be the canonical weak* continuous extension. This is RCP, e.g. by an argument in the proof of [4, Theorem 2.6]. The restriction to $W=X^{* *}$ is the canonical weak* continuous extension $\hat{T}: X^{* *} \rightarrow B(H)$. Then

$$
\left\|\widehat{T}_{c}\right\|_{\mathrm{cb}}=\left\|\widehat{T}_{c}\right\|=\left\|\widehat{T}_{c}(1)\right\|=\|\hat{T}(1)\| .
$$

We may then apply the unital case to $T^{* *}$ (resp. $\hat{T}$ ). For example the canonical extension of this weak* continuous map to $W+W^{*}$ is (selfadjoint and) completely positive. Restricting to $X+X^{*}$ we see that the same is true for the canonical extension $\tilde{T}$ to $X+X^{*}$. We have

$$
\|T\| \leq\|\tilde{T}\| \leq\|\tilde{T}\|_{\mathrm{cb}} \leq\left\|\widehat{T}_{c}\right\|_{\mathrm{cb}}=\|\hat{T}(1)\| \leq \sup _{t}\left\|T\left(e_{t}\right)\right\| \leq\|T\|,
$$

for any J-cai $\left(e_{t}\right)$ for $A$. Thus these are all equal. A similar argument works with $\hat{T}$ replaced by $T^{* *}$. We show that this number equals $\lim _{t}\left\|T\left(e_{t}\right)\right\|$. Indeed if a subnet of $\left(\left\|T\left(e_{t}\right)\right\|\right)$ had limit $<\|T\|$, then by a further replacement we may suppose all terms in this subnet were bounded above by $\alpha<\|T\|$. Replacing $\left(e_{t}\right)$ by the appropriate subnet in the last centered equation would yield the contradiction $\|T\| \leq \alpha<\|T\|$. 
Corollary 2.5. If $T: X \rightarrow B(H)$ is a unital map on a unital real operator space then $T$ is completely contractive if and only if $T$ is real completely positive.

Proof. The $(\Leftarrow)$ direction follows from Proposition 2.4. The other direction can be seen for example by going to the complexification and then using the complex case of the present result (see e.g. [4).

By Proposition 2.4 if $X$ is a real unital operator space then $X+X^{*}$ is well defined as a real operator system. Indeed if $T: X \rightarrow Y$ is a surjective unital complete isometry between unital operator spaces $X \subset B(H)$ and $Y \subset B(K)$, then $T$ is real completely positive. The canonical extension $\tilde{T}: X+X^{*} \rightarrow B(K)$ : $x+y^{*} \mapsto T(x)+T(y)^{*}$ is selfadjoint and is a completely isometric complete order embedding onto $Y+Y^{*}$. As in the proof this may also be seen by extending to the complexification. The operator space $X+X^{*}$ in $B(H)$ has the operator space complexification $\left(X+X^{*}\right)_{c} \subset B\left(H_{c}\right)$. In addition, $X_{c}+X_{c}^{*}$ is a completely reasonable operator space complexification of $X+X^{*}$. By the uniqueness of the operator space complexification (see the introduction), $X_{c}+X_{c}^{*}=\left(X+X^{*}\right)_{c}$.

Theorem 2.6. If $\pi: A \rightarrow B$ is a homomorphism between real $C^{*}$-algebras, or a Jordan homomorphism between real $J C^{*}$-algebras, then $\pi$ is contractive if and only if $\pi$ is selfadjoint (hence is a *-homomorphism or Jordan *-homomorphism). In this case $\pi$ is positive, indeed completely positive and completely contractive in the real $C^{*}$-algebra case.

Proof. Assume that $\pi$ is contractive. By taking biduals we may assume that $A$ is a real $W^{*}$-algebra and $B=B(H)$. For any projection $p \in A$ we have that $\pi(p)$ is a contractive idempotent, hence is an orthogonal projection. In particular, $\pi(1)$ is a projection. Replacing $H$ by $\pi(1) H, \pi$ becomes unital. If $x=x^{*}$ then by the spectral theorem we may approximate $x$ by real linear combinations of projections. Using this and the fact about projections proved at the start of the proof, we see that $\pi(x)$ is selfadjoint. Suppose that $x^{*}=-x$ and that $\varphi$ is a real state on $B$. Then $\varphi \circ \pi$ is a real state on $A$, and so $\varphi(\pi(x))=0$. Thus $\pi(x)$ is antisymmetric by [23. Exercise 14A] (see also Lemma 2.1.19 in [44). Thus $\pi$ is selfadjoint by Lemma 2.2 .

If $\pi$ is a Jordan $*$-homomorphism then $\pi_{c}: A_{c} \rightarrow B_{c}$ is a Jordan $*$-homomorphism. By the corresponding fact for complex Jordan $C^{*}$-algebras, $\pi_{c}$ is contractive and positive. Thus, $\pi_{\mid A}=\pi$ is contractive and positive. (The positivity can also be seen more directly by the spectral theorem as in the last paragraph.)

Let $A$ and $B$ be real $C^{*}$-algebras. If $\pi: A \rightarrow B$ is a contractive homomorphism, then by the above $\pi$ is a $*$-homomorphism. Then $\pi_{c}: A_{c} \rightarrow B_{c}$ is a *-homomorphism. Thus, $\pi_{c}$ is completely positive and completely contractive by a fact in complex $C^{*}$-algebras. Since $\left.\pi_{c}\right|_{A}=\pi, \pi$ is completely positive and completely contractive.

The following is an analog of the Stinespring dilation and the Arveson extension theorem for completely positive maps on real unital Jordan operator algebras.

Theorem 2.7. Let $A$ be a unital subspace or real approximately unital Jordan subalgebra of a real $C^{*}$-algebra $B$ and let $T: A \rightarrow B(H)$ be a real completely positive map. Then $T$ has a completely positive extension $\tilde{T}: B \rightarrow B(H)$. In addition there is a $*$-representation $\pi: B \rightarrow B(K)$ for a real Hilbert space $K$, and 
a contraction $V \in B(H, K)$, such that

$$
\tilde{T}(a)=V^{*} \pi(a) V, \quad a \in B .
$$

Moreover, this can be done with $\|T\|=\|T\|_{\mathrm{cb}}=\|\tilde{T}\|_{\mathrm{cb}}=\|V\|^{2}$, and this equals $\|T(1)\|$ if $A$ is unital.

Proof. By Proposition $2.4 T$ is completely bounded, with $\|T\|_{\mathrm{cb}}=\|T\|$. If $A$ is unital then by Proposition $2.4 T$ has a unital completely positive extension to $A+$ $A^{*}$, and we may extend further by [40, Proposition 4.2] to a selfadjoint completely positive map $\tilde{T}: B \rightarrow B(H)$, of cb norm $\|T(1)\|$. If $A$ is nonunital let $W=A^{* *}$. By the proof of Proposition 2.4 the canonical extension $u=\widetilde{\hat{T}}$ of $\hat{T}$ to $W+W^{*}$ is selfadjoint and completely positive, and has the same cb norm. We may extend further by [40, Proposition 4.2] to a selfadjoint completely positive map $B^{* *} \rightarrow$ $B(H)$, of cb norm $\|\hat{T}(1)\|$. Let $\tilde{T}$ be the restriction to $B$.

By Theorem 4.3 in [40], there is a $*$-representation $\pi: B \rightarrow B(K)$ where $K$ is a real Hilbert space and bounded operator $V \in B(H, K)$ such that $\tilde{T}(a)=V^{*} \pi(a) V$ for all $a \in B$, and $\|V\|^{2}=\|\hat{T}(1)\|=\|T\|_{\mathrm{cb}}=\|T\|$.

Corollary 2.8. A real positive linear functional on a unital real subspace or approximately unital real Jordan subalgebra of a real $C^{*}$-algebra $B$, extends to a positive selfadjoint functional on $B$ with the same norm.

This follows e.g. from the last theorem and Lemma 5.14 or can be seen more directly e.g. as in the proof of Lemma 5.14. Indeed the functionals in the last result are just the positive multiples of states.

Let $X$ and $Y$ be operator spaces. If $T: X \rightarrow Y$ is a completely bounded, then $\|T\|_{\mathrm{cb}}=\left\|T_{c}\right\|_{\mathrm{cb}}$ by [41, Theorem 2.1]. However this is not true at the 'Banach level', if $T: X \rightarrow Y$ is contractive, then $T_{c}$ may not be contractive. This depends on the operator space structures that are given to $X$ and $Y$, as we shall now see.

Example 2.9. Let $X$ and $Y$ be $l_{2}^{1}(\mathbb{R})$ with the maximal and minimal operator space structures from [42] respectively, and let $T: X \rightarrow Y$ be the identity map, a complete contraction. One obtains a complete contraction $T_{c}: X_{c} \rightarrow Y_{c}$. One can easily show that $Y_{c}$ may be identified completely isometrically with the two dimensional complex $C^{*}$-algebra $l_{2}^{\infty}(\mathbb{C})$ (since $l_{2}^{1}(\mathbb{R}) \cong l_{2}^{\infty}(\mathbb{R})$ isometrically). On the other hand, $X_{c}$ is $l_{2}^{1}(\mathbb{C})$ with the maximal operator space structure (which is known to equal its minimal operator space structure). To see this note that by Propositions 2.6 and 2.3 in $\left[42\right.$, and by the fact above, we have that $\left(\operatorname{Max}\left(l_{2}^{1}(\mathbb{R})\right)\right)_{c}$ equals

$$
\left(\left(\operatorname{Min}\left(l_{2}^{\infty}(\mathbb{R})\right)\right)^{*}\right)_{c}=\left(\left(\operatorname{Min}\left(l_{2}^{\infty}(\mathbb{R})\right)\right)_{c}\right)^{*}=l_{2}^{\infty}(\mathbb{C})^{*}=\operatorname{Max}\left(l_{2}^{1}(\mathbb{C})\right) .
$$

We also used the duality of Min and Max for complex operator spaces [7, Section 1.4].

Thus $T_{c}$ cannot be an isometry or complete isometry, since it is well known that $l_{2}^{1}(\mathbb{C})$ and $l_{2}^{\infty}(\mathbb{C})$ are not isometrically isomorphic. Indeed $\left(T^{-1}\right)_{c}$ cannot be a contraction, even though $u=T^{-1}$ is an isometry.

Proposition 2.10. There exist real unital operator algebras $A$ and $B$ with operator algebra complexifications $A_{c}$ and $B_{c}$, and a contractive (even isometric) unital homomorphism $\theta: A \rightarrow B$ whose complexification $\theta_{c}: A_{c} \rightarrow B_{c}$ is not contractive. 
Proof. Let $X$ and $Y$ be as in Example 2.9 above. We may view $X \subset B(H)$ and let $B=\mathcal{U}(X)$ be the set of 'upper triangular' matrices

$$
a=\left[\begin{array}{cc}
\alpha I_{H} & x \\
0 & \beta I_{H}
\end{array}\right]
$$

where $\alpha, \beta \in \mathbb{R}$ and $x \in X \subseteq B(H)$. Note that $\mathcal{U}(X)$ is a real unital operator algebra, and is a subspace of the real Paulsen system $\mathcal{S}(X)=\mathcal{U}(X)+\mathcal{U}(X)^{*}$ (see [42, Lemma 4.12] and the lines above it). We claim that $\mathcal{U}(X)_{c}=\mathcal{U}\left(X_{c}\right)$ and $\mathcal{S}(X)_{c}=$ $\mathcal{S}\left(X_{c}\right)$ (these complexifications are the unique operator space complexifications). Indeed this claim follow easily from the facts that $M_{2}(B(H))_{c}=M_{2}\left(B(H)_{c}\right)=$ $M_{2}\left(B\left(H_{c}\right)\right.$ ), and $\mathcal{S}(X) \subset \mathcal{S}\left(X_{c}\right) \subset M_{2}\left(B(H)_{c}\right)$. Following the proof of Proposition 2.2.11 in [7], we obtain that

$$
\|a\|^{2}=\sup \left\{\left(|\alpha| \sqrt{1-t^{2}}+\|x\| t\right)^{2}+|\beta t|^{2}: t \in[0,1]\right\} .
$$

From this equation, we can easily see that

$$
\left\|\left[\begin{array}{cc}
\alpha & x \\
0 & \beta
\end{array}\right]\right\|=\left\|\left[\begin{array}{cc}
|\alpha| & \|x\| \\
0 & |\beta|
\end{array}\right]\right\| .
$$

Similarly $A=\mathcal{U}(Y)$ is an operator algebra. By the last norm formula the isometry $u: Y \rightarrow X$ in Example 2.9 extends to an isometric unital homomorphism $\theta_{u}: A \rightarrow B$. However suppose that $\theta_{u}$ extended to a contractive unital map $r$ on $\mathcal{U}(Y)_{c}=\mathcal{U}\left(Y_{c}\right)$. Then $r$ would be real positive, and hence by e.g. the proof of [10, Lemma 2.1] it would extend further to a positive selfadjoint unital map on $\mathcal{S}\left(Y_{c}\right)$. By e.g. (1.25) in [7] this forces the 1-2-corner map $Y_{c} \rightarrow X_{c}$ to be contractive. However this map is $u_{c}$, giving a contradiction.

Many results in the theory of complex operator algebras involving completely contractive maps will be almost identical in the real case. For example, Corollary 2.3 and Corollary 4.18 of 9 or Theorem 2.5 of 8 concerning completely contractive projections $P: A \rightarrow A$ on an operator algebra or Jordan operator algebra, will be true in the real case. This follows quickly by applying the complex case of these results to $P_{c}$. Similarly for Banach-Stone theorems characterizing complete isometries between operator algebras or Jordan operator algebras (such as [10, Theorem 3.5] (2) (note $C=B$ there if $B$ is also an operator algebra, by (1)) or [8, Proposition 6.5] or [7, Theorem 4.5.13]). See e.g. 411, Theorem 4.4].

In passing we mention the Kadison-Banach-Stone theorem for real $J C^{*}$-algebras (see e.g. [28, Theorem 4.8] and [20]): A surjective linear map $T: A \rightarrow B$ between real $J C^{*}$-algebras is an isometry if and only if $T$ is a 'triple morphism' (that is, preserves the natural 'triple product'). In addition if these hold then $T$ is a Jordan homomorphism if and only if it is positive. We sketch a proof of the last assertion: note that by Theorem 2.6, a contractive Jordan homomorphism is selfadjoint and positive. Conversely, if $T$ is a positive isometry then so is $T^{* *}$. This uses the Kaplansky density theorem for real $J C^{*}$-algebras, which may be proved following a standard proof for the complex case of that result. Then $u=T^{* *}(1)$ is positive and also is a partial isometry, indeed is a unitary in $B^{* *}$ in the $J W^{*}$-algebra sense, since $T^{* *}$ is a triple morphism. Hence $u=\left(u^{2}\right)^{\frac{1}{2}}=1$. The triple morphism property then implies that $T$ is a Jordan homomorphism. We do not know if there is a variant of the Banach-Stone theorems above or in [10 for surjective isometries or surjective real positive isometries between e.g. unital real Jordan operator algebras. 
Finally we mention some results on the $C^{*}$-envelope and injective envelope, some of benefitted from discussions with Mehrdad Kalantar and which we hope to present elsewhere. There is a difficulty here that we overcome which is related to injective envelopes of dynamical systems. For the $C^{*}$-envelope and injective envelope in the complex case we refer to [35, Chapter 15] or [7, Chapter 4], or the papers of Hamana and Ruan referenced there. A preliminary study of the injective envelope and $C^{*}$-envelope in the real case may be found in [42. Using notation from those sources we are able to prove:

Theorem 2.11. Let $A$ be a unital real operator space or operator system, or if $A$ is an approximately unital real operator algebra (or Jordan operator algebra). Then $I(A)_{c}=I\left(A_{c}\right)$. Also, $I(A)$ is a unital real $C^{*}$-subalgebra of $I\left(A_{c}\right)$, and if $C_{e}^{*}(A)$ is the $C^{*}$-subalgebra of $I(A)$ generated by $A$ then $C_{e}^{*}(A)_{c}=C_{e}^{*}\left(A_{c}\right)$.

In this result, $C_{e}^{*}(\mathcal{S})$ has the universal property of the $C^{*}$-envelope: given any unital complete isometry $j: \mathcal{S} \rightarrow D$ into a real $C^{*}$-algebra $D$ such that $j(\mathcal{S})$ generates $D$ as a real $C^{*}$-algebra, there exists a -epimorphism $\pi: D \rightarrow C_{e}^{*}(\mathcal{S})$ such that $\pi \circ j$ is the canonical inclusion of $\mathcal{S}$ in $C_{e}^{*}(\mathcal{S})$.

There is a result analogous to $I(\mathcal{S})_{c}=I\left(\mathcal{S}_{c}\right)$ in the context of Hamana's $G$ injective envelope [24, 25]. Namely that $I_{G}(\mathcal{S})=I(\mathcal{S})$ for a finite group $G$ and an operator system $\mathcal{S}$ which is a $G$-module in the sense of Hamana [24, 25]. A similar result holds for the $G$ - $C^{*}$-envelope. The case of this where $G=\mathbb{Z}_{2}$ was the inspiration for the last proof. We hope to present this elsewhere in work with Mehrdad Kalantar and a graduate student.

\section{Unitization (MEYeR's THEOREM)}

In [42, Theorem 3.5] a real variant of Meyer's unitization theorem was proved for completely contractive homomorphisms on real operator algebras. Namely any completely contractive real linear homomorphism $A \rightarrow B(K)$ on a subalgebra $A$ of $B(H)$ not containing $I_{H}$, extends to a unital completely contractive real linear homomorphism $A+\mathbb{R} I_{H} \rightarrow B(K)$. This implies that the unitization of a real operator algebra is uniquely defined up to completely isometric algebra isomorphism. The variant of Meyer's theorem for contractive $\mathbb{C}$-linear homomorphisms on complex Jordan operator algebras was noted in [16. However it is more difficult to prove the real version of the latter result, and we turn to this next.

Lemma 3.1. Let $A \subseteq B(H)$ be a real (Jordan) operator algebra and $A_{c} \subseteq B\left(H_{c}\right)$ be its complexification where $H$ is a real Hilbert space. Assume that $I_{H} \notin A$. Then for $a, b \in A$ and $\lambda \in \mathbb{C}$, we have

$$
|\lambda| \leq\left\|(a+i b)+\lambda I_{H}\right\|
$$

Proof. We may replace $A_{c}$ by the closed algebra generated by $a+i b$. Then this follows from [7, Lemma 2.1.12].

Theorem 3.2 (Meyer type unitization). Let $A$ be a real subalgebra (resp. Jordan subalgebra) of $B(H)$, and assume that $I_{H} \notin A$. Let $\pi: A \rightarrow B(K)$ be a contractive homomorphism (resp. Jordan homomorphism) for a real Hilbert space $K$. Let $A^{1}=$ $\operatorname{span}_{\mathbb{R}}\left\{A, I_{H}\right\} \subseteq B(H)$ and define $\pi^{o}: A^{1} \rightarrow B(K)$ by $\pi^{o}\left(a+\lambda I_{H}\right)=\pi(a)+\lambda I_{K}$. Then $\pi^{0}$ is a contractive homomorphism (resp. contractive Jordan homomorphism). 
Proof. We follow the proof of Meyer's theorem for a complex operator algebra (see Theorem 2.1.13 in [7]) using the fact that $A$ has a complexification which is a complex operator algebra.

It is easy to see that $\pi^{0}$ is a homomorphism (resp. Jordan homomorphism). To show that it is contractive, let $T=a+\lambda I_{H} \in A^{1}$ for some $a \in A$ and $\lambda \in \mathbb{R}$ and $\|T\|<1$. We may effectively replace $A$ by the closed algebra generated by $a$, which is an operator algebra. We claim that $\left\|\pi^{\circ}(T)\right\|<1$. We will regard everything as objects inside $B\left(H_{c}\right)$. In particular we view $A, A^{1}=A+\mathbb{R} I_{H}$, and $B(H)$ as real subalgebras of $B\left(H_{c}\right)$, and we view $T$ as an operator in $B\left(H_{c}\right)$. By Lemma 3.1 $|\lambda|<1$. Since $T$ is strictly contractive, by item (2) in 2.1 .14 in 7 we have that $(I+T)(I-T)^{-1}$ is strictly accretive. Set $\alpha=(1+\lambda) /(1-\lambda)$. Then $\alpha>0$ and

$$
\theta=\frac{1}{\alpha}(I+T)(I-T)^{-1}=I+\frac{1}{\alpha}\left((I+T)(I-T)^{-1}-(I+\lambda)(I-\lambda)^{-1}\right)
$$

is also strictly accretive. By the Neumann lemma, $(I-T)^{-1}=\sum_{k=0}^{\infty} T^{k}$, which lies in $A+\mathbb{R} I$. We may write $(I+T)(I-T)^{-1}-(I+\lambda)(I-\lambda)^{-1}$ as

$$
(I-T)^{-1}((I+T)(I-\lambda)-(I-T)(I+\lambda))(I-\lambda)^{-1},
$$

which equals $2(I-T)^{-1} a(I-\lambda)^{-1}=\frac{2}{1-\lambda}(I-T)^{-1} a$. Since $A$ is an ideal in $A+\mathbb{R} I$, we have

$$
\theta-I=\alpha^{-1}(I+T)(I-T)^{-1}-(I+\lambda)(I-\lambda)^{-1} \in A .
$$

Since $\theta$ is accretive, $\theta+I$ is invertible. By the principle $A \cap\left(A_{c}\right)^{-1}=A^{-1}$ mentioned in the introduction, we have $(\theta+I)^{-1} \in A+\mathbb{R} 1$. So we must have $(\theta-I)(\theta+I)^{-1} \in$ $A$, again since $A$ is an ideal of $A+\mathbb{R} 1$.

Since $\pi_{c}^{o}$ is a unital homomorphism and $\theta+I$ is invertible, $\pi_{c}^{o}(\theta+I)=\pi_{c}^{o}(\theta)+I$ is invertible and $\left.\pi_{c}\left((\theta+I)^{-1}\right)=\left(\pi_{c}^{o}(\theta)+I\right)\right)^{-1}$. Thus,

$$
\pi_{c}^{o}\left((\theta-I)(\theta+I)^{-1}\right)=\left(\pi_{c}^{0}(\theta)-I\right)\left(\pi_{c}^{o}(\theta)+I\right)^{-1} .
$$

We will use items (1) and (2) in 2.1.14 in [7] several times. We know that $\theta$ is strictly accretive, thus $(\theta-I)(\theta+I)^{-1}$ is strictly contractive and is an element in $A \subseteq A_{c}$. Since $\left.\pi_{c}^{o}\right|_{A}=\pi, \pi_{c}^{\circ}\left((\theta-I)(\theta+I)^{-1}\right) \in B(K)$. Since $\pi$ is a contraction, $\left\|\left(\pi_{c}^{0}(\theta)-I\right)\left(\pi_{c}^{o}(\theta)+I\right)^{-1}\right\|_{B\left(K_{c}\right)}$ equals

$$
\left\|\pi_{c}^{\circ}\left((\theta-I)(\theta+I)^{-1}\right)\right\|_{B\left(K_{c}\right)}=\left\|\pi\left((\theta-I)(\theta+I)^{-1}\right)\right\|_{B(K)}<1 .
$$

Thus, $\pi_{c}^{o}(\theta)$ is strictly accretive in $B\left(K_{c}\right)$. Thus,

$$
\alpha \pi_{c}^{0}(\theta)=\pi_{c}^{o}\left((I+T)(I-T)^{-1}\right)=\left(I+\pi_{c}^{o}(T)\right)\left(I-\pi_{c}^{o}(T)\right)^{-1}
$$

is strictly accretive. Therefore $\pi_{c}^{o}(T)=\pi^{\circ}(T)$ is strictly contractive as desired.

It now follows that the unitization of a real operator algebra is unique up to isometric isomorphism:

Theorem 3.3. Let $A$ be a real subalgebra (resp. Jordan subalgebra) of $B(H)$, and assume that $I_{H} \notin A$. Let $\pi: A \rightarrow B(K)$ be an isometric homomorphism (resp. isometric Jordan homomorphism) for a real Hilbert space $K$. Then the unital homomorphism $\pi^{\circ}: A^{1} \rightarrow B(K)$ where $\pi^{\circ}\left(a+\lambda I_{H}\right)=\pi(a)+\lambda I_{K}$ is an isometric isomorphism onto $\pi(A)+\mathbb{R} I_{H}$.

Proof. This follows from Theorem 3.2 as in [7, Corollary 2.1.15]. 
Corollary 3.4. The unitization $A^{1}$ of a Jordan operator algebra is unique up to isometric Jordan isomorphism. In addition, $\left(A^{1}\right)_{c}=\left(A_{c}\right)^{1}$ isometrically isomorphically.

Proof. We follow the proof of Corollary 2.5 in [16. If $A$ is nonunital then we may assume that $A$ is represented on a Hilbert space $H$ and the Jordan operator algebra unitization $A^{1}$ of $A$ is identified with $A+\mathbb{R} I_{H}$. Then the first assertion follows from Theorem 3.3. If $A$ is unital and $e$ is the identity of $A$, then $e$ is a central projection of a unitization $A^{1}$. Also $e$ commutes with $A^{*}$ (adjoints with respect to a fixed unital isometric representation of $A^{1}$; this follows for example since $e$ is selfadjoint in that representation by the statement about $\Delta(A)$ in Lemma 5.7). So $e$ is central in $C^{*}\left(A^{1}\right)$. From this it is easy to see that for $a \in A, \lambda \in \mathbb{R}$ we have

$$
\|a+\lambda 1\|=\max \{\|e(a+\lambda 1)\|,\|(1-e)(a+\lambda 1)\|\}=\max \{\|a+\lambda e\|,|\lambda|\} .
$$

Since a unitization of complex Jordan operator algebra is unique up to isometric Jordan homomorphism (see [16, Corollary 2.5]), $\left(A^{1}\right)_{c}=\left(A_{c}\right)^{1}$.

\section{Approximate identities}

If $A$ is a Jordan subalgebra of a $C^{*}$-algebra $B$ (either real or complex case) then we say that a net $\left(e_{t}\right)$ in $\operatorname{Ball}(A)$ is a $B$-relative partial cai for $A$ if $e_{t} a \rightarrow a$ and $a e_{t} \rightarrow a$ for all $a \in A$. Here we are using the usual product on $B$, which may not give an element in $A$, and may depend on $B$. We say that a net $\left(e_{t}\right)$ in $\operatorname{Ball}(A)$ is a partial cai for $A$ if for every $C^{*}$-algebra $B$ containing $A$ as a Jordan subalgebra, $e_{t} a \rightarrow a$ and $a e_{t} \rightarrow a$ for all $a \in A$, using the product on $B$. Note that partial cais are the same as cais if $A$ is an associative operator algebra. We say that $A$ is approximately unital if it has a partial cai. If $A$ is an operator algebra or Jordan operator algebra then we recall that a net $\left(e_{t}\right)$ in $\operatorname{Ball}(A)$ is a Jordan cai or $J$-cai for $A$ if $e_{t} a+a e_{t} \rightarrow 2 a$ for all $a \in A$.

Lemma 4.1. Let $A$ be a real Jordan subalgebra of a real $C^{*}$-algebra $B$. Then

(1) A has a $B$-relative partial cai if and only if $A_{c}$ has a $B_{c}$-relative partial cai.

(2) A has a J-cai if and only if $A_{c}$ has a J-cai.

(3) If $A_{c}$ has a partial cai then $A$ has a partial cai.

Proof. Note that $A_{c}$ is a complex Jordan subalgebra of the $C^{*}$-algebra $B_{c}$. Any $B$-relative partial cai of $A$ is a $B_{c}$-relative partial cai of $A_{c}$. Conversely, if $\left(e_{t}+i f_{t}\right)$ is a $B_{c}$-partial cai of $A_{c}$, then $\left(e_{t}\right)$ is a $B$-partial cai of $A$. (See the fact in the proof of [31, Proposition 5.2.4].) Similarly for J-cai's. Item (3) follows from the ideas in (1).

Lemma 4.2. If $A$ is a real Jordan subalgebra of a real $C^{*}$-algebra $B$, then the following are equivalent:

(i) A has a partial cai.

(ii) A has a B-relative partial cai.

(iii) A has a J-cai.

(iv) $A^{* *}$ has an identity $p$ of norm 1 with respect to the Jordan Arens product on $A^{* *}$, which coincides on $A^{* *}$ with the restriction of the usual product in $B^{* *}$. Indeed $p$ is the identity of the von Neumann algebra $C_{B}^{*}(A)^{* *}$. 
If these hold then any partial cai $\left(e_{t}\right)$ for $A$ is a cai for $C_{B}^{*}(A)$ (and for the associative operator algebra generated by $A$ ), and every $J$-cai for $A$ converges weak* ${ }^{*}$ to $p$.

Proof. This holds almost exactly as in the complex case [16, Lemma 2.6]. We just indicate a proof that (iv) implies (i). Suppose that $p$ is an identity for $A^{* *}$. Viewing $A$ as with its operator space structure we have that $\left(A_{c}\right)^{* *}=\left(A^{* *}\right)_{c}$ by 41. Since the canonical map $A_{c} \rightarrow\left(A^{* *}\right)_{c}$ is a Jordan homomorphism, so is its weak ${ }^{*}$ continuous extension $\left(A_{c}\right)^{* *} \rightarrow\left(A^{* *}\right)_{c}$. Thus $\left(A_{c}\right)^{* *}=\left(A^{* *}\right)_{c}$ as dual real Jordan operator algebras. Thus $p$ is the identity of $\left(A_{c}\right)^{* *}$. By the complex case of the present result, $A_{c}$ has a partial cai $\left(e_{t}+i f_{t}\right)$, with $e_{t}, f_{t} \in A$. Therefore by the proof of the last lemma $\left(e_{t}\right)$ is a partial cai for $A$.

Proposition 4.3. Let $A$ be an approximately unital real Jordan operator algebra and let $\pi: A \rightarrow B(H)$ be a contractive Jordan homomorphism. We let $P$ be the projection onto $K=[\pi(A) H]$. Then $\pi\left(e_{t}\right) \rightarrow P$ in the weak* (and WOT) topology of $B(H)$ for any $J$-cai $\left(e_{t}\right)$ for $A$. Moreover, for $a \in A$, we have $\pi(a)=P \pi(a) P$, and the compression of $\pi$ to $K$ is a contractive Jordan homomorphism. Also, if $\left(e_{t}\right)$ is a partial cai for $A$, then $\pi\left(e_{t}\right) \pi(a) \rightarrow \pi(a)$ and $\pi(a) \pi\left(e_{t}\right) \rightarrow \pi(a)$. In particular, $\left.\pi\left(e_{t}\right)\right|_{K} \rightarrow I_{K}$ SOT in $B(K)$.

Proof. As in the proof of Lemma 2.19 in [16].

We will see in the proof of the next theorem that if $\left(a_{t}+i b_{t}\right)$ is a cai for $A_{c}$ in $\frac{1}{2} \mathfrak{F}_{A_{c}}$ (resp. $\mathfrak{r}_{A_{c}}$ ) then $\left(a_{t}\right)$ is a cai for $A$ in $\frac{1}{2} \mathfrak{F}_{A}$ (resp. $\mathfrak{r}_{A}$ ).

Theorem 4.4 (Real case of Theorem 2.8 of [16]). If $A$ is an approximately unital real Jordan operator algebra then $\mathfrak{F}_{A}$ is weak $k^{*}$ dense in $\mathfrak{F}_{A^{* *}}$ and $\mathfrak{r}_{A}$ is weak dense in $\mathfrak{r}_{A^{* *}}$. Finally, $A$ has a partial cai in $\frac{1}{2} \mathfrak{F}_{A}$.

Proof. Let $\left(A_{c},\|\cdot\|_{c}\right)$ be the operator space complexification of $A$. Then $\mathfrak{F}_{A_{c}}$ is weak* dense in $\mathfrak{F}_{A_{c}^{* *}}$. Let $x \in \mathfrak{F}_{A^{* *}} \subset \mathfrak{F}_{A_{c}^{* *}}$. By the density in the complex case, there is a net $\left(a_{t}+i b_{t}\right)$ weak ${ }^{*}$ converging to $x$, which implies that $a_{t}$ weak $^{*}$ converges to $x$. Since $\left\|a_{t}-1\right\| \leq\left\|a_{t}+i b_{t}-1\right\|_{c} \leq 1$, we have $a_{t} \in \mathfrak{F}_{A}$. This shows that $\mathfrak{F}_{A}$ is weak* dense in $\mathfrak{F}_{A^{* *}}$.

Similarly, if $x \in \mathfrak{r}_{A^{* *}} \subset \mathfrak{r}_{A_{c}^{* *}}$ then there is a net $\left(a_{t}+i b_{t}\right)$ in $\mathfrak{r}_{A_{c}}$ weak* converging to $x$. Since $\left(a_{t}+i b_{t}\right)+\left(a_{t}+i b_{t}\right)^{*} \geq 0$, we have $a_{t}+a_{t}^{*} \geq 0$. Moreover, $a_{t}$ weak* converges to $x$.

Finally, by the corresponding fact in the complex case, $A_{c}$ has a partial cai $\left(e_{t}+\right.$ $\left.i f_{t}\right)$ in $\frac{1}{2} \mathfrak{r}_{A_{c}}$. Thus, $\left(e_{t}\right)$ is a partial cai in $A$. Since $\left\|1-\frac{1}{2} e_{t}\right\| \leq\left\|1-\frac{1}{2}\left(e_{t}+i f_{t}\right)\right\| \leq 1$, we have that $e_{t} \in \frac{1}{2} \mathfrak{r}_{A}$.

A similar proof gives the analogue of Proposition 2.10 and Corollary 2.11 of [16]:

Proposition 4.5. Let $A$ be an approximately unital real Jordan operator algebra. Then the set of contractions in $\mathfrak{r}_{A}$ is weak ${ }^{*}$ dense in the set of contractions in $\mathfrak{r}_{A^{* *}}$.

Proposition 4.6. If $A$ is a Jordan operator algebra with a countable Jordan cai, then $A$ has a countable partial cai in $\frac{1}{2} \mathfrak{F}_{A}$.

In the following result, which generalizes [16, Theorem 4.1] (which in turn derives from [14, Theorem 2.1]), we write $x \preccurlyeq y$ to denote $\operatorname{Re}(x) \leq \operatorname{Re}(y)$. Here $\operatorname{Re}(x)=$ $\left(x+x^{*}\right) / 2$. Also $\mathfrak{c}_{A}=\mathbb{R}^{+} \mathfrak{F}_{A}$. 
Theorem 4.7. Let $A$ be a real Jordan operator algebra which generates a real $C^{*}$ algebra $B$, and let $\mathcal{U}_{A}$ denote the open unit ball $\{a \in A:\|a\|<1\}$. The following are equivalent:

(1) A is approximately unital.

(2) For any positive $b \in \mathcal{U}_{B}$ there exists $a \in \mathfrak{r}_{A}$ with $b \preccurlyeq a$.

(2') Same as (2), but $a \in \frac{1}{2} \mathfrak{F}_{A}$.

(3) For any pair $x, y \in \mathcal{U}_{A}$ there exist $a \in \frac{1}{2} \mathfrak{F}_{A}$ with $x \preccurlyeq a$ and $y \preccurlyeq a$.

(4) For any $b \in \mathcal{U}_{A}$ there exist $a \in \frac{1}{2} \mathfrak{F}_{A}$ with $-a \preccurlyeq b \preccurlyeq a$.

(5) For any $b \in \mathcal{U}_{A}$ there exist $x, y \in \frac{1}{2} \mathfrak{F}_{A}$ with $b=x-y$.

(6) $\mathfrak{r}_{A}$ is a generating cone (that is, $A=\mathfrak{r}_{A}-\mathfrak{r}_{A}$ ).

(7) $A=\mathfrak{c}_{A}-\mathfrak{c}_{A}$.

Proof. (1) $\Rightarrow\left(2^{\prime}\right)$ Let $A$ be an approximately unital real Jordan operator algebra, and $b \in A_{+}$with $\|b\|<1$. Then $A_{c}$ is approximately unital, and by [16, Theorem 4.1 (2)] there exists $x+i y \in \frac{1}{2} \mathfrak{F}_{A_{c}}$ such that $b \leq \operatorname{Re}(x+i y)$. This is easily seen to imply that $b \leq \operatorname{Re}(x)$. Since $\|1-2 x\| \leq\|1-2 x-2 i y\| \leq 1$ we have $x \in \frac{1}{2} \mathfrak{F}_{A}$.

The other implications are as in [14, Theorem 2.1] and [16, Theorem 4.1], however some of the results invoked in those proofs need to be replaced by their real variants from the present paper. Also we note that the implications $\left(2^{\prime}\right) \Rightarrow(3)$ and $(2) \Rightarrow$ (6) follow from a fact from $C^{*}$-algebra theory. Namely, from the Claim: if $x$ and $y$ (with $y=-x$ in the second implication) are selfadjoint and in $\mathcal{U}_{B}$ then there is a positive element in $\mathcal{U}_{B}$ which is greater than both. This is true in the real case too. To see this we may assume that $x$ and $y$ are also in $B_{+}$, by replacing them by $x_{+}$and $y_{+}$. In this case the Claim is usually an ingredient in standard proofs that $B$ has an increasing cai. However this case of the Claim also follows from the same fact but in the complex case. Indeed there exists $a+i b \in B_{c}$ with (i) $\|a+i b\|<1$, and (ii) $x$ and $y$ dominated by $a+i b$. However (i) implies that $\|a\|<1$ and (ii) implies that $x$ and $y$ are dominated by $a$.

A Jordan ideal in a Jordan operator algebra $A$ is a subspace $J$ of $A$ with $J \circ A \subset J$.

Proposition 4.8. Let $A$ be an approximately unital real operator algebra (resp. Jordan operator algebra).

(1) If A is weak* closed then the weak* closed ideals (resp. Jordan ideals) in A which possess an identity are in a bijective correspondence with the central projections $e \in A$, via $e \mapsto A e$ (resp. $e \mapsto e \circ A$ ). These ideals are $M$ summands of $A$.

(2) The closed ideals (resp. Jordan ideals) in A which possess a cai (resp. J-cai) are the subspaces of $A$ whose weak* closure in $A^{* *}$ are of the form in (1) for a central projection $e \in A^{* *}$. Thus they are of form $\{x \in A: x=e x e\}$ for some such $e \in A^{* *}$.

Proof. This follows just as in the complex case in e.g. [16, Theorem 3.25].

We will do a more thorough study elsewhere of the $M$-ideals in real Jordan operator algebras (following on from [42, Section 5]).

Corollary 4.9. If $J$ is an approximately unital closed two-sided ideal (resp. Jordan ideal) in a real operator algebra (resp. Jordan operator algebra) A, then $A / J$ is (completely isometrically isomorphic to) a real operator algebra (resp. Jordan operator algebra). 
Proof. This follows from Proposition 4.8 just as in the complex case in e.g. [16, Theorem 3.27].

Turning to one-sided ideals, Sharma showed in [42, Section 5] that the closed right ideals in an approximately unital real operator algebra $A$ which possess a left cai are the subspaces of $A$ whose weak* closure in $A^{* *}$ are of the form $e A^{* *}$ for a projection $e \in A^{* *}$. Thus they are of form $\{x \in A: x=e x\}$ for some such $e \in A^{* *}$. The projections $e$ occurring here are called open projections. The corresponding subspace $\{x \in A: x=e x e\}$ is called a hereditary subalgebra of $A$. For a real Jordan operator algebra $A$ we define a hereditary subalgebra to be a subspace of $A$ whose weak* closure in $A^{* *}$ is of the form $e A^{* *} e$ for a projection $e \in A^{* *}$. Thus they are of form $\{x \in A: x=e x e\}$ for such $e \in A^{* *}$. We will discuss elsewhere the noncommutative topology and hereditary subalgebras of real Jordan operator algebras, in the spirit of 9 .

Lemma 4.10. If $A$ is a nonunital approximately unital real Jordan operator algebra then the unitization $A^{1}$ is well defined up to completely isometric Jordan isomorphism, and the matrix norms are

$$
\left\|\left[a_{i j}+\lambda_{i j} 1\right]\right\|=\sup \left\{\left\|\left[a_{i j} \circ c+\lambda_{i j} c\right]\right\|_{M_{n}(A)}: c \in \operatorname{Ball}(A)\right\}, \quad a_{i j} \in A, \lambda_{i j} \in \mathbb{R} .
$$

Proof. The proof is the same as for the complex case in Proposition 2.12 in [16].

Remark 4.11. A unitization of a real Jordan operator algebra $A$ need not be well defined/unique up to completely isometric Jordan homomorphism. This may be seen by modifying the argument as in Proposition 2.1 in [17] (see [44, Remark 4.4.3]). This means that some results about real Jordan operator algebras with no kind of approximate identity may not be treatable in the operator space category (as opposed to the Banach space category). If however $A$ is approximately unital then the last result shows that this problem does not exist.

As in the complex case [16, Theorem 2.8], a real approximately unital Jordan operator algebra $A$ is an $M$-ideal in $A^{1}$.

Lemma 4.12 (Real case of Lemma 2.20 in 16]). Let $A$ be a real approximately unital Jordan operator algebra with a partial cai $\left(e_{t}\right)$. Denote the identity of $A^{1}$ by 1. The following facts hold.

(1) If $\psi: A^{1} \rightarrow \mathbb{R}$ is a functional on $A^{1}$, then $\lim _{t} \psi\left(e_{t}\right)=\psi(1)$ if and only if $\|\psi\|=\left\|\left.\psi\right|_{A}\right\|$.

(2) Let $\varphi \in A^{*}$. Then $\varphi$ uniquely extends to a functional on $A^{1}$ of the same norm.

Proof. (1) We have $\left(A^{1}\right)_{c}=\left(A_{c}\right)^{1}$ by Corollary 3.4. If $\psi: A^{1} \rightarrow \mathbb{R}$ is a functional on $A^{1}$, then $\|\psi\|=\left\|\psi_{c}\right\|$ by Proposition 1.4.1 in 31. By Lemma 4.1, $\left(e_{t}\right)$ is a partial cai for $A_{c}$. By Lemma 2.20 in [16], $\lim _{t} \psi\left(e_{t}\right)=\psi(1)$ if and only if $\left\|\psi_{c}\right\|=\left\|\left(\psi_{c}\right)_{\mid A_{c}}\right\|$. Now $\left(\psi_{c}\right)_{\mid A_{c}}=\left(\psi_{\mid A}\right)_{c}$, and so

$$
\left\|\left(\psi_{c}\right)_{\mid A_{c}}\right\|=\left\|\left(\psi_{\mid A}\right)_{c}\right\|=\left\|\psi_{\mid A}\right\|
$$

by Proposition 1.4.1 in 31. Thus $\lim _{t} \psi\left(e_{t}\right)=\psi(1)$ if and only if $\|\psi\|=\left\|\left.\psi\right|_{A}\right\|$.

(2) This uses a similar idea: if $\psi, \rho$ are two Hahn-Banach extensions of $\varphi$ to $A^{1}$, then $\psi_{c}, \rho_{c}$ are two Hahn-Banach extensions of $\varphi_{c}$ to $A_{c}^{1}$, by Proposition 1.4.1 in 31. 
Lemma 4.13 (Real case of Lemma 2.21 in [16]). For a norm 1 functional $\varphi$ on an approximately unital real Jordan operator algebra A, the following are equivalent:

(1) $\varphi$ extends to a state on $A^{1}$.

(2) $\varphi\left(e_{t}\right) \rightarrow 1$ for every partial cai $e_{t} \in A$.

(3) $\varphi\left(e_{t}\right) \rightarrow 1$ for some partial cai for $A$.

(4) $\varphi(e)=1$ where $e$ is the identity of $A^{* *}$.

(5) $\varphi\left(e_{t}\right) \rightarrow 1$ for every Jordan cai for $A$.

(6) $\varphi\left(e_{t}\right) \rightarrow 1$ for some Jordan cai for $A$.

Proof. The proof is the same as for the complex case in Lemma 2.21 in [16].

The functionals on $A$ characterized in the last lemma are the states of $A$.

\section{ReAl POSItive ELEMENTS AND REAL POSITIVE MAPS}

As we said in the introduction, if $A$ is a unital subspace or unital (Jordan) subalgebra of $B(H)$ then then the set $\mathfrak{r}_{A}$ of real positive elements in $A$ does not depend on the particular $B(H)$ that $A$ sits in (isometrically and unitally). A similar statement holds if $A$ is any (Jordan) subalgebra of $B(H)$. Thus if $A$ is a Jordan operator algebra and $\pi: A \rightarrow B(K)$ is an isometric Jordan homomorphism then, for example, $\pi(x)+\pi(x)^{*} \geq 0$ if and only if e.g. $\|1-t x\| \leq 1+t^{2}\|x\|^{2}$ for all $t>0$. Here 1 is the identity of $A^{1}$, or the identity operator on $H$. This is a simple consequence of Meyer's theorem for Jordan operator algebras above. Hence $A \cap \mathfrak{r}_{A_{c}}=\mathfrak{r}_{A}$ if $A_{c}$ is any Jordan operator algebra complexification of $A$. Similarly it is clear from Meyer's theorem above for Jordan operator algebras that $\mathfrak{F}_{A}=A \cap \mathfrak{F}_{A_{c}}$.

In the following proof we write $\operatorname{Re}(x)$ for $\frac{1}{2}\left(x+x^{*}\right)$.

Lemma 5.1. If $X$ is a real unital operator space or Jordan operator algebra then $\mathfrak{r}_{X}=\overline{\mathbb{R}^{+} \mathfrak{F}_{X}}$.

Proof. If $\|1-x\| \leq 1$ then $\|1-\operatorname{Re}(x)\| \leq 1$. Therefore $-1 \leq 1-\operatorname{Re}(x) \leq 1$, and so $\operatorname{Re}(x) \geq 0$. Thus $\overline{\mathbb{R}^{+} \mathfrak{F}_{X}} \subset \mathfrak{r}_{X}$. The reverse inclusion in the unital operator space case can be proved as is done in the complex case early in [4, Section 2]. If $X$ is a Jordan operator algebra then $\mathfrak{r}_{X} \subset \mathfrak{r}_{X_{c}}=\overline{\mathbb{R}^{+} \mathfrak{F}_{X_{c}}}$. Suppose that $x \in \mathfrak{r}_{X}$ and $c_{t} x_{t} \rightarrow x$ with $c_{t} \in \mathbb{R}^{+}$and $x_{t}=a_{t}+i b_{t} \in \mathfrak{F}_{X_{c}}$. Then $a_{t} \in X \cap \mathfrak{F}_{X_{c}}=\mathfrak{F}_{X}$, and $c_{t} a_{t} \rightarrow x$. So $x \in \overline{\mathbb{R}^{+} \mathfrak{F}_{X}}$.

Remark 5.2. As in [14, 16] we may consider the $\mathfrak{F}$-transform: By [14, Lemma 2.5], if $x \in \mathfrak{r}_{A}$ for a real Jordan operator algebra $A$ then

$$
\mathfrak{F}(x)=x(x+1)^{-1} \in A \cap \frac{1}{2} \mathfrak{F}_{A_{c}}=\frac{1}{2} \mathfrak{F}_{A} .
$$

Indeed let $D$ be the real operator algebra generated by 1 and $x$. Since $x+x^{*} \geq 0$, the numerical range of $x$ is in the right half plane. Hence the spectrum in $D_{c}$ of $x$ is in the right half plane. Hence -1 is not in that spectrum, so $1+x$ has an inverse in $D_{c}$, in fact in $D$ (since e.g. if $x(a+i b)=1$ then $x a=1$ ). Then $\mathfrak{F}(x)=x(1+x)^{-1} \in A D \subset A$. Also, $1-2 x(1+x)^{-1}=(1-x)(1+x)^{-1}$, which is essentially the Cayley transform, which has norm $\leq 1$. In fact this map has range $U_{A} \cap \frac{1}{2} \mathfrak{F}_{A}$, where $U_{A}=\{a \in A:\|a\|<1\}$, as in [14, Lemma 2.5]. Indeed suppose that $w \in U_{A} \cap \frac{1}{2} \mathfrak{F}_{A} \subset U_{A_{c}} \cap \frac{1}{2} \mathfrak{F}_{A_{c}}$. We may suppose that $A$ is the closed algebra generated by $w$, which is an operator algebra. Then there exists $x \in \mathfrak{r}_{A_{c}}$ 
with $\mathfrak{F}(x)=w$. However $x=w(1-w)^{-1} \in A$. Using the $\mathfrak{F}$-transform one may give another proof of Lemma 5.1 in the spirit of e.g. [13, Theorem 3.3].

Lemma 5.3. Let $A$ be an operator system or real $J C^{*}$-algebra. Then $x \in A$ is antisymmetric if and only if $x \in \mathfrak{r}_{A} \cap\left(-\mathfrak{r}_{A}\right)$.

This is useful because there are many nice metric characterizations of $\mathfrak{r}_{A}$, as we said earlier when we defined that set (for example the conditions in [5, Lemma $2.4])$.

Lemma 5.4. If $X$ is a real operator system then $X=X_{\mathrm{sa}} \oplus X_{\mathrm{as}}$. Also, $X_{\mathrm{sa}}=$ $X_{+}-X_{+}$, and $X=\mathfrak{r}_{X}-\mathfrak{r}_{X}=\mathbb{R}^{+}\left(\mathfrak{F}_{X}-\mathfrak{F}_{X}\right)$.

Proof. The first identity was established above Lemma 2.2. Let $x \in X_{\mathrm{sa}}$, then

$$
x=\frac{1}{2}(\|x\| 1+x-(\|x\| 1-x)) \in X_{+}-X_{+} .
$$

Similarly, since $\|x\| 1-(\|x\| 1 \pm x)$ has norm $\leq\|x\|$, we have that $\|x\| 1 \pm x \in\|x\| \mathfrak{F}_{X} \subset$ $\mathbb{R}^{+} \mathfrak{F}_{X}$. The rest is clear.

If $X$ is a real operator system and $T: X \rightarrow B(H)$ we say that $T$ is systematically real positive if $x, y \in X$ with $x+y^{*} \in X_{+}$implies that $T(x)+T(y)^{*} \geq 0$.

Theorem 5.5. Let $X$ be a real selfadjoint operator space and let $T: X \rightarrow B(H)$ be real linear. The following are equivalent:

(i) $T$ is systematically real positive.

(ii) $T$ is both positive and selfadjoint.

(iii) $T$ is real positive and selfadjoint.

Proof. Clearly a selfadjoint map is positive if and only if it is real positive, and if these holds then $T: X \rightarrow B(H)$ is systematically real positive. Conversely suppose that $T$ is systematically real positive. Let $x \in X_{+}$. Then $T(x)+T(0)^{*} \geq 0$, so $T$ is positive. By Lemma 5.4 we have $T\left(X_{\mathrm{sa}}\right)=T\left(X_{+}\right)-T\left(X_{+}\right) \subset B(H)_{\mathrm{sa}}$. If $x^{*}=-x$ then $x+x^{*}=0$, so that $T(x)+T(x)^{*}$ is both positive and negative. Hence $T(x)^{*}=-T(x)$. That is, $T\left(X_{\text {as }}\right) \subset Y_{\text {as }}$. So $T$ is selfadjoint by Lemma 2.2.

Theorem 5.6. If $T: A \rightarrow B(H)$ is a real positive linear map on a unital operator space $A$ whose restriction to $\Delta(A)=A \cap A^{*}$ is selfadjoint (or systematically real positive). Then the canonical extension $\tilde{T}: A+A^{*} \rightarrow B(H): x+y^{*} \mapsto T(x)+T(y)^{*}$ is well defined, selfadjoint, and positive.

Proof. Let $T: A \rightarrow B(H)$ be real positive with $T$ restricted to $\Delta(A)$ being selfadjoint. Define $\tilde{T}\left(a+b^{*}\right)=T(a)+T(b)^{*}$ for $a, b \in A$. To see that $\tilde{T}$ is well defined, suppose $a+b^{*}=x+y^{*}$, for $a, b, x, y \in A$. Then $a-x=(y-b)^{*} \in \Delta(A)$, and so

$$
T(a-x)=T\left((y-b)^{*}\right)=(T(y)-T(b))^{*} .
$$

Thus, $T$ is well defined. If $z=a+b^{*}$ is positive (usual sense), then

$$
z=z^{*}=b+a^{*}=\frac{1}{2}\left(a+b^{*}+b+a^{*}\right)=\frac{1}{2}(a+b)+\left(\frac{1}{2}(a+b)\right)^{*},
$$

and $\frac{1}{2}(a+b) \in \mathfrak{r}_{A}$. Since $T$ is real positive we have

$$
\tilde{T}(z)=T\left(\frac{1}{2}(a+b)\right)+T\left(\frac{1}{2}(a+b)\right)^{*} \geq 0 .
$$

So $\tilde{T}$ is positive. 
The converse of the theorem is true: if $\tilde{T}$ is well defined, selfadjoint, and positive, then $T$ is real positive and its restriction to $\Delta(A)=A \cap A^{*}$ is selfadjoint and systematically real positive.

If $X$ is a unital real operator space then we say that a real linear map $T$ : $X \rightarrow B(H)$ is systematically real positive if $T$ extends to a positive selfadjoint map on $X+X^{*}$. This is equivalent, by the theorem, to $T$ being real positive with $T$ restricted to $\Delta(A)$ being selfadjoint. It is also equivalent to: $x, y \in X$ with $x+y^{*} \geq 0$ implies that $T(x)+T(y)^{*} \geq 0$. One way to see the last equivalence is to note that this condition implies that $T$ restricted to $\Delta(A)$ is systematically real positive, hence selfadjoint by Theorem 5.5. Then apply the last theorem.

Note that a real positive linear map from an operator system $X$ into $B(H)_{\text {sa }}$ is systematically real positive. Indeed in this case $T\left(X_{\mathrm{sa}}\right) \subset B(H)_{\text {sa }}$, and as in the proof of Theorem 5.5 above $T\left(X_{\text {as }}\right) \subset(0) \subset B(H)_{\text {sa }}$. So $T$ is selfadjoint by Lemma 2.2 .

We discuss now the meaning of $A+A^{*}$ for a real operator algebra or real Jordan operator algebra $A$. If $A$ is also equipped with a compatible operator space structure (that is, if $A$ is a Jordan subalgebra of $B(H)$ and has the inherited matrix norms), then this is relatively unproblematic. This is essentially the case treated in 44]. The point is that a completely isometric (Jordan) homomorphism $\theta: A \rightarrow B$ between real (Jordan) operator algebras extends to a completely isometric (Jordan) homomorphism between their complexifications. By the complex theory this extends to a completely isometric (Jordan) homomorphism between the unitizations of the complexifications, and then to a completely isometric UCP map $\left(A_{c}\right)^{1}+\left(\left(A_{c}\right)^{1}\right)^{*} \rightarrow\left(B_{c}\right)^{1}+\left(\left(B_{c}\right)^{1}\right)^{*}$. This restricts to a completely isometric selfadjoint complete order isomorphism $A+A^{*} \rightarrow B+B^{*}$.

On the other hand, if we treat $A$ without using matrix norms, that is, use morphisms that are isometric (Jordan) homomorphisms, then it seems that the meaning of $A+A^{*}$ is more problematic. That is, we do not know at present if an isometric (Jordan) homomorphism $\theta: A \rightarrow B$ extends to an isometric selfadjoint order isomorphism $A+A^{*} \rightarrow B+B^{*}$. However we will know shortly from Lemma 5.7 that it extends to a selfadjoint order isomorphism $\tilde{\theta}: A+A^{*} \rightarrow B+B^{*}$. There is a canonical norm on $A+A^{*}$ for which the latter map is an isometry (namely the norm inherited from $C_{\max }^{*}(A)$, the universal $C^{*}$-algebra for contractive (Jordan) homomorphisms from $A$, see 44]), but we do not know yet if this norm always agrees with the one mentioned above in the last paragraph using a suitable operator space structure on $A$. (It does follow from the later result Lemma 5.19 that $\tilde{\theta}$ is isometric on the selfadjoint part of $A+A^{*}$.) In any case, if we concern ourselves only with the order structure on $A+A^{*}$ there are no problems.

Lemma 5.7. Let $\theta: A \rightarrow B$ be a contractive (Jordan) homomorphism between real (Jordan) operator algebras. Then $\theta$ extends uniquely to a selfadjoint positive map $\tilde{\theta}: A+A^{*} \rightarrow B+B^{*}$. Also, restriction of $\theta$ to $\Delta(A)$ is a (Jordan) *-homomorphism into $\Delta(B)$. If $\theta$ is also an isometric isomorphism onto $B$ then $\tilde{\theta}$ is a selfadjoint order isomorphism onto $B+B^{*}$.

Proof. We know that $\Delta(A)$ is a real $J C^{*}$-algebra. Suppose that $B$ is a Jordan subalgebra of $B(H)$. By Theorem 2.6, the restriction of $\theta$ to $\Delta(A)$ is a Jordan *-homomorphism into $B(H)$. In particular it is selfadjoint, and maps into $\Delta(B)$. The proof of Theorem 5.6 now gives the first assertion. Alternatively, one may extend $\theta$ to a unital contractive (Jordan) homomorphism between unitizations of 
$A$ and $B$ by Theorem 3.2, and then appeal to the statement of Theorem 5.6. The second assertion follows from the first applied to $\theta$ and $\theta^{-1}$.

For a real operator algebra or real Jordan operator algebra $A$ then we say that a real linear map $T: A \rightarrow B(H)$ is systematically real positive if $T$ is real positive with $T$ restricted to $\Delta(A)=A \cap A^{*}$ being selfadjoint. It is also equivalent to the canonical extension $\tilde{T}: A+A^{*} \rightarrow B(H): x+y^{*} \mapsto T(x)+T(y)^{*}$ being well defined, selfadjoint, and positive. Indeed the proof of Theorem 5.6 shows that if $T$ is real positive with $T$ restricted to $\Delta(A)$ selfadjoint then $\tilde{T}$ is well defined, selfadjoint, and positive. Conversely, if the last condition holds then clearly $T$ is real positive and $T$ restricted to $\Delta(A)=A \cap A^{*}$ is selfadjoint. The latter implies that $x+y^{*} \geq 0$ implies that $T(x)+T(y)^{*} \geq 0$, but we are not sure if this condition is equivalent.

Remark 5.8. Another class of maps that one could consider are the maps $T: A \rightarrow B$ that extend to a real positive map on a (Jordan) operator algebra complexification. Then of course there are various variants of this class, such as contractions that extend to a real positive contraction on such a complexification.

Lemma 5.9. Let $T: A \rightarrow B$ be a systematically real positive map between real Jordan operator algebras. Then $T(\Delta(A)) \subset \Delta(B)$, and $T$ restricts to a positive selfadjoint linear map from $\Delta(A)$ to $\Delta(B)$. Thus $0 \leq T(1) \leq 1$ if $A$ is unital and $T$ is contractive.

Proof. As above, $\tilde{T}: A+A^{*} \rightarrow B(H)$ is positive and selfadjoint. Hence so is its restriction to $\Delta(A)$.

Let $T: X \rightarrow B(H)$ be a unital linear contraction on a unital operator space. Then $T$ is real positive in the sense that $T$ takes $\mathfrak{r}_{X}$ to real positive operators. This follows from the fact that $\mathfrak{r}_{X}=\overline{\mathbb{R}^{+} \mathfrak{F}_{X}}$ (see Lemma 5.1). However we shall see that $T$ need not be systematically real positive. Indeed if $X$ is a real operator system then $T$ need not be selfadjoint, although $T$ is antisymmetric (that is, $T\left(X_{\text {as }}\right) \subset Y_{\text {as }}$ ). Indeed if $x^{*}=-x$ then $x+x^{*}=0$, so that $T(x)+T(x)^{*}$ is both positive and negative. Hence $T(x)^{*}=-T(x)$.

Example 5.10. Let $X$ be the polynomials with real coefficients of degree $\leq 1$ in $C([0,1])$. Let $x(t)=t$, and let $z$ be the matching monomial in the disk algebra. Let $g=\frac{1+z}{2}$, a contraction in the disk algebra, and define $T(s+t x)=s+t g$ for $s, t \in \mathbb{R}$. We claim that $T$ is a unital contraction on $X$ which is not selfadjoint, nor systematically real positive, nor positive. Indeed the norm of $s+t g$ in the disk algebra is easily seen to be $|s+t / 2|+|t| / 2$, whereas it is an exercise that the norm of $s+t x$ in $X$ is $\max \{|s|,|s+t|\}$. Finally, by considering the cases that $s, t$, and $s+t / 2$ are positive and negative, one can prove easily that

$$
|s+t / 2|+|t| / 2 \leq \max \{|s|,|s+t|\}, \quad s, t \in \mathbb{R} .
$$

That is, $T$ is a unital contraction on $X$. It clearly is not selfadjoint, hence is not systematically real positive.

This example illustrates some other points. First, although $T$ is a unital contraction on $X$, and although $X$ is so very simple, $T$ is not a complete contraction. Indeed if it were then by [41, Theorem 2.1] it extends to a unital complete contraction on the complexification. This extension would have to be selfadjoint by the well known complex case [3, 35, giving the contradiction that $T$ is selfadjoint. Also, there is not a real version of the completely contractive version of von 
Neumann's inequality (sometimes attributed to Sz-Nagy, and following from the Sz-Nagy dilation), else $T$ would be completely contractive. There is a real form of von Neumann's inequality. (Indeed if $T$ is a contraction in $B(H) \subset B(H)_{c}$ then the map $p+\bar{q} \mapsto p(T)+q(T)^{*}$ is a positive (hence completely positive, by [35. Theorem 3.11]) unital contraction from a dense selfadjoint subspace of $C(\mathbb{T}, \mathbb{C})$ into $B(H)_{c}$. The restriction to the set of $p+\bar{q}$ for polynomials $p, q$ with real coefficients, is a positive selfadjoint contraction into $B(H)$.)

Note that $R(s+t x)=T(s+t x) \oplus(s+t x) \in A(\mathbb{D}) \oplus^{\infty} X$ is a unital isometry which is not systematically real positive, nor selfadjoint, nor positive. Thus if $X$ is a unital operator space, $X+X^{*}$ need not be 'well defined' as an ordered Banach space. Indeed in the above example $X$ and $T(X)$ are 'isometrically the same' as unital operator spaces via the unital isometry $T$. However $X+X^{*}$ has dimension 2 , while $T(X)+T(X)^{*}$ has dimension 3 , so $T$ certainly does not extend to a faithful map on $X+X^{*}$, let alone a positive selfadjoint isometric one, nor an order isomorphism onto its range. On the other hand $X+X^{*}$ is 'well defined' as an ordered linear space if we use morphisms on a unital operator space $X$ that are real positive unital isometries which is selfadjoint on $\Delta(X)$. Indeed if $T: X \rightarrow Y$ is a surjective unital isometry between unital operator spaces $X$ and $Y$, and if $T$ and $T^{-1}$ are systematically real positive, then the canonical extension $\tilde{T}: A+A^{*} \rightarrow B(H): x+y^{*} \mapsto T(x)+T(y)^{*}$ is selfadjoint and an order embedding. Also, $X+X^{*}$ is 'well defined' as an operator system, if we use morphisms on a unital operator space $X$ that are unital complete isometries. See the remark above Theorem 2.6 .

Example 5.11. A real positive map, or systematically real positive map, need not extend to a real positive map on a complexification. A positive selfadjoint map on a real operator system need not extend to a positive map on a complexification. Also, a unital linear contraction need not extend to a real positive map on a fixed complexification. For an example of these, we proceed as in Proposition 2.10, Let $u: Y \rightarrow X$ be a linear contraction that does not extend to a contraction from $Y_{c}$ to $X_{c}$. Let $\theta_{u}$ be the canonical extension of $u$ to a unital contractive homomorphism $\mathcal{U}(Y) \rightarrow \mathcal{U}(X)$. As we said in Proposition 2.10, by Theorem 5.6, or by the real variant of the Paulsen lemma in [42, Lemma 4.12] (see also p. 492 in [40]), $\theta_{u}$ extends to a positive selfadjoint map $\theta_{u}+\left(\theta_{u}\right)^{*}$ on the Paulsen system $\mathcal{S}(Y)=\mathcal{U}(Y)+\mathcal{U}(Y)^{*}$ (which is even real contractive by Lemma 5.19). Thus $\theta_{u}$ is systematically real positive. But it does not extend to a real positive map on the complexification, by the argument in Proposition 2.10,

Example 5.12. A positive functional even on a real $C^{*}$-algebra need not be selfadjoint nor real positive. The example above Proposition 4.1 in [40] shows this: apply that functional to the real positive matrix with all entries 1 except for a -3 in the 1-2 corner. Note that if we scale this example to be a unital functional $\psi$, then $\|\psi\| \geq 1$. But in fact $\|\psi\|>1$ since if $\|\psi\|=1$ then $\psi$ would be selfadjoint by the next result.

Example 5.13. A positive unital selfadjoint real linear map on a complex operator system need not be a contraction. There is a $2 \times 2$ matrix counterexample due to Arveson see e.g. A.2 in [3]. Viewing this as a real operator system this map is unital and real positive, but not a contraction.

Indeed a positive unital selfadjoint real linear map on a real $J C^{*}$-algebra need not be bounded. For an example of this let $E$ be an infinite dimensional space 
of selfadjoint operators on a Hilbert space $H$ with $x^{2} \in \mathbb{R} I_{H}$ for all $x \in E$. Let $A$ be the set of matrices in $M_{2}(B(H))$ with diagonal entries in $\mathbb{R} I_{H}$, and offdiagonal entries $x$ and $-x$ for $x \in E$. Note that $A$ is a real $J C^{*}$-algebra. We have $A_{\text {sa }}=\left(\mathbb{R} I_{H}\right) \oplus\left(\mathbb{R} I_{H}\right)$, the positive elements in $A$ are $\left(\mathbb{R}_{+} I_{H}\right) \oplus\left(\mathbb{R}_{+} I_{H}\right)$, and $A_{\text {as }}$ consists of the matrices in $A$ with zero main diagonal entries. If $T: E \rightarrow B(K)_{\text {sa }}$ is an unbounded real linear map let $\theta_{T}$ be the map on $A$ taking

$$
\left[\begin{array}{cc}
\lambda I & x \\
-x & \mu I
\end{array}\right] \mapsto\left[\begin{array}{cc}
\lambda I & T x \\
-T x & \mu I
\end{array}\right]
$$

This is a positive unital selfadjoint real linear map on a real $J C^{*}$-algebra which is not bounded. If $K=\mathbb{R}$ in Example 5.13 then $\theta_{T}: A \rightarrow M_{2}$. This positive map is not 2-positive, otherwise by Lemma 5.17 it would be completely positive, and hence completely contractive by Lemma 2.3 .

Lemma 5.14. For a functional $\varphi$ on a unital real operator space or approximately unital real Jordan operator algebra $X$ the following are equivalent:

(i) $\varphi$ is real positive.

(ii) $\varphi$ is systematically real positive.

(iii) $\varphi$ is real completely positive (RCP).

(iv) $\varphi$ is a nonnegative multiple of a state.

Such functionals are bounded with $\|\varphi\|=\|\varphi\|_{\mathrm{cb}}$. This equals $\varphi(1)$, or $\lim _{t} \varphi\left(e_{t}\right)$ in the case of a cai $\left(e_{t}\right)$. If $\varphi$ is unital then the above equivalent conditions hold iff $\varphi$ is contractive. If $X$ is a real operator system then a unital functional $\varphi$ on $X$ is contractive if and only if it is positive and selfadjoint; such a functional is completely positive.

Proof. Suppose that $\varphi$ is a real positive functional on a unital real operator space $X$. Its restriction to $\Delta(X)=X \cap X^{*}$ is a real positive functional on an operator system. If $x^{*}=-x$ in $\Delta(X)$ then as in the proof of Theorem 5.5 we have that $\varphi(x)=0$. So $\varphi$ is selfadjoint on $\Delta(X)$ by Lemma 2.2 Hence $\varphi$ is systematically real positive by Theorem [5.6. Such maps are real completely positive, as in the complex case. One way to see this is if $\tilde{\varphi}$ is the canonical extension to $X+X^{*}$, and if $x=\left[x_{i j}\right] \geq 0$ in $M_{n}\left(X+X^{*}\right)$ then $\left[\tilde{\varphi}\left(x_{i j}\right)\right]$ is a selfadjoint matrix. We have

$$
\left\langle\left[\tilde{\varphi}\left(x_{i j}\right)\right] \xi, \xi\right\rangle=\tilde{\varphi}\left(\xi^{T} x \xi\right) \geq 0 .
$$

So $\left[\tilde{\varphi}\left(x_{i j}\right)\right] \geq 0$. So $\tilde{\varphi}$ is completely positive and hence $\varphi$ is real completely positive. Since $\tilde{\varphi}$ is completely positive it extends to a completely positive, hence completely bounded, map on the complexification (see e.g. Proposition 2.4). Indeed the norm and completely bounded norm of this extension is $\varphi(1)$ by e.g. [35, Proposition 3.6], hence $\|\varphi\|=\|\varphi\|_{\mathrm{cb}}=\varphi(1)$.

If $A$ is an approximately unital real Jordan operator algebra then $A=\mathfrak{r}_{A}-\mathfrak{r}_{A}$ by Theorem 4.7. If $\varphi$ is a real positive functional then the argument of [14, Corollary $2.8]$ shows that $\varphi$ is bounded. Hence $\varphi^{* *}$ is real positive, and by the above $\varphi^{* *}$ is systematically real positive and real completely positive (RCP), with $\left\|\varphi^{* *}\right\|=$ $\left\|\varphi^{* *}\right\|_{\mathrm{cb}}=\varphi^{* *}(1)$. Hence $\|\varphi\|=\|\varphi\|_{\mathrm{cb}}=\lim _{t} \varphi\left(e_{t}\right)$, the latter since $e_{t} \rightarrow 1$ weak* $^{*}$ for a cai $\left(e_{t}\right)$.

A contractive unital functional on a unital real operator space $X$ extends to a contractive unital functional on a real $C^{*}$-algebra. This is positive and selfadjoint by [31, Proposition 5.2.6 (3)], and so its restriction is systematically real positive, and indeed positive if $X$ is an operator system. 
In particular any state on a unital real operator space is real positive. Similarly on an approximately unital real Jordan operator algebra (e.g. by taking the bidual and using the same argument). Conversely, if $\varphi$ is real positive and nontrivial then $\frac{1}{\alpha} \varphi$ is a state, where $\alpha$ is $\varphi(1)$, or $\lim _{t} \varphi\left(e_{t}\right)$ in the case of a cai $\left(e_{t}\right)$.

A selfadjoint functional on an operator system is clearly real positive if and only if it is positive, and is a positive multiple of a state. So if it is unital it is a state and has norm 1. Such maps are real completely positive and completely positive, as in the complex case-see e.g. the argument a few paragraphs above.

Remark 5.15. If $A$ is a real $J C^{*}$-algebra then a positive and selfadjoint functional is real positive, systematically real positive, and completely positive, and is RCP.

A unital linear contraction on a unital operator space is real positive as was stated above. However the converse is false in general, see Example 5.13. Nonetheless, we have:

Lemma 5.16. For a real linear map $T: X \rightarrow B$ from a unital real operator space or approximately unital real Jordan operator algebra into a commutative real $C^{*}$-algebra the following are equivalent:

(i) $T$ is real positive.

(ii) $T$ is systematically real positive.

(iii) $T$ is real completely positive.

Such maps are bounded with $\|T\|=\|T\|_{\mathrm{cb}}$. This equals $\|T(1)\|$, or $\lim _{t}\left\|T\left(e_{t}\right)\right\|$ in the case that $A$ has a J-cai $\left(e_{t}\right)$. If $T$ is unital then the above hold iff $T$ is contractive. If $X$ is a real operator system and $T$ is unital then $T$ is contractive if and only if it is positive and selfadjoint; such a map is completely positive.

Proof. This follows from Lemma 5.14 and the usual trick for this in the complex case. Indeed the commutative real $C^{*}$-algebra may be replaced by $C(K, \mathbb{C})$ viewed as a real *-algebra by basic facts about commutative real $C^{*}$-algebras (see [39, Theorem 1.9] or [31]). Then apply Lemma 5.14 to the linear functional $\psi_{w}=$ $T(\cdot)(w)$ for fixed $w \in K$. This will be real positive if $T$ is real positive, and so $\left\|\psi_{w}\right\|=\psi_{w}(1)$ by Lemma 5.14. Thus $\|T\|$ equals

$$
\sup \{|T(f)(w)|: w \in K, f \in \operatorname{Ball}(X)\}=\sup \{|T(1)(w)|: w \in K, f \in \operatorname{Ball}(X)\}
$$

which is $\|T(1)\|$. In particular $T$ is bounded. For an approximately unital real Jordan operator algebra $A$ we can take a weak* continuous extension on $A^{* *}$ to reduce to the unital case as in e.g. Proposition 2.4 .

If $T$ is a unital contraction then $T$ is real positive as we said above Example 5.10. If further $X$ is a real operator system then $T$ is positive and selfadjoint by (ii). It is also completely contractive since $\|T\|=\|T\|_{\mathrm{cb}}$, so applying the above to each $T_{n}$ we see that it is completely positive.

Lemma 5.17. Let $T: A \rightarrow M_{n}$ be a linear map on a unital operator space, or a bounded linear map on an approximately unital real Jordan operator algebra, which is real $n$-positive (that is, $T_{n}$ is real positive). Then $T$ is $R C P$ and systematically real positive, and $\|T\|_{\mathrm{cb}}=\|T\|$. The latter equals $\|T(1)\|$ if $A$ is unital, otherwise equals $\left\|T^{* *}(1)\right\|=\lim _{t}\left\|T\left(e_{t}\right)\right\|$, if $\left(e_{t}\right)$ is a J-cai for $A$.

Proof. Suppose that $x=\left[x_{i j}\right]$ and $x+x^{*} \geq 0$ in $M_{m}\left(A+A^{*}\right)$. Then $\left[T\left(x_{i j}\right)\right]+$ $\left[T\left(x_{j i}\right)^{*}\right]=T_{m}(x)+T_{m}(x)^{*}$ is certainly selfadjoint. So to test if this is positive it is enough to check that $\left\langle\left(T_{m}(x)+T_{m}(x)^{*}\right) \eta, \eta\right\rangle \geq 0$ for $\eta \in\left(\mathbb{R}^{n}\right)^{m}$ and $m>n$. 
However in a real Hilbert space $\left\langle\left(z+z^{*}\right) \eta, \eta\right\rangle=2\langle z \eta, \eta\rangle$. Hence it is enough to check that $\left\langle T_{m}(x) \eta, \eta\right\rangle \geq 0$. As in the proof of Proposition 2.2.2 in [22] there is an isometry $\alpha: \mathbb{R}^{n} \rightarrow \mathbb{R}^{m}$ and $\xi \in\left(\mathbb{R}^{n}\right)^{n}$ such that $\eta=\left(\alpha \otimes I_{n}\right)(\xi)$. Set $y=\alpha^{*} x \alpha$ then $y \in M_{n}(A)$ and

$$
2\left\langle T_{n}(y) \xi, \xi\right\rangle=\left\langle\left(T_{n}(y)+T_{n}(y)^{*}\right) \xi, \xi\right\rangle \geq 0
$$

since $T_{n}$ is real positive. Hence as in Proposition 2.2.2 in [22],

$$
\left\langle T_{m}(x) \eta, \eta\right\rangle=\left\langle T_{n}\left(\alpha^{*}\left[x_{i j}\right] \alpha\right) \xi, \xi\right\rangle=\left\langle T_{n}(y) \xi, \xi\right\rangle \geq 0 .
$$

Thus $T$ is RCP. The rest follows from Proposition 2.4

Remark 5.18. There is a Schwarz inequality for 2-positive real linear maps on real $C^{*}$-algebras, proved identically to e.g. [35, Proposition 3.3]. See p. 492 in [40, for the Schwarz inequality for real UCP maps.

The map Re $: B(H) \rightarrow B(H)_{\text {sa }}$ is real positive, positive, unital, contractive and selfadjoint.

We say that a map $u: X \rightarrow Y$ is real contractive if $\|\operatorname{Re} u(x)\| \leq\|\operatorname{Re} x\|$ for $x \in X$. We say that $u$ is real bounded if there is a constant $c \geq 0$ with $\|\operatorname{Re} u(x)\| \leq c\|\operatorname{Re} x\|$ for $x \in X$, and then we write $\|u\|_{r}$ for the least $c$ in this inequality. This is called the real bounded norm (actually it is a seminorm).

Lemma 5.19. Let $A$ be a real unital operator space. If $u: A \rightarrow B(H)$ is real positive and restricts to selfadjoint map on $\Delta(A)$ then $u$ is real bounded with $\|u\|_{r}=$ $\|u(1)\|$. Indeed $u$ extends to a positive selfadjoint map $\tilde{u}: \mathcal{S}=A+A^{*} \rightarrow B(H)$, with $\|\tilde{u}\|_{r}=\|u(1)\|$.

If $A$ is an approximately unital real Jordan operator algebra and $u: A \rightarrow B(H)$ is real positive and restricts to selfadjoint map on $\Delta(A)$ then $u$ is real bounded with $\|u\|_{r}=\sup _{t}\left\|u\left(e_{t}\right)\right\|$, where $\left(e_{t}\right)$ is any Jordan cai for $A$.

Proof. First assume $A$ is unital. The restriction of $u$ to $\Delta(A)$ is real positive, so by Theorem 5.6 we have that $u$ is systematically real positive, $u(1) \geq 0$, and $u$ extends to a positive selfadjoint $\tilde{u}: \mathcal{S}=A+A^{*} \rightarrow B(H)$. For any unit vector $\xi \in H$, by Lemma 5.14 we have that $\varphi_{\xi}=\langle u(x) \xi, \xi\rangle$ is systematically real positive and bounded with norm $\langle u(1) \xi, \xi\rangle$. By the proof of Lemma 5.14. $\varphi_{\xi}$ extends to a positive selfadjoint functional $\psi_{\xi}$ on $\mathcal{S}$ of norm $\langle u(1) \xi, \xi\rangle$.

For $x \in \operatorname{Ball}(\mathcal{S})$ we have $\|\operatorname{Re} x\| \leq\|x\| \leq 1$. We have $\operatorname{Re} \tilde{u}(x)=\tilde{u}(\operatorname{Re} x)$ and $\|\operatorname{Re} \tilde{u}(x)\|$ equals

$$
\sup |\langle\tilde{u}(\operatorname{Re} x) \xi, \xi\rangle|=\sup \left|\psi_{\xi}(\operatorname{Re} x)\right|
$$

(suprema over $\xi \in H,\|\xi\|=1$ ). This is dominated by $\sup \langle u(1) \xi, \xi\rangle=\|u(1)\|$.

Next, if $A$ is an approximately unital real Jordan operator algebra then the same argument gives that $\varphi_{\xi}$ is systematically real positive and bounded with norm $\sup _{t}\left|\left\langle u\left(e_{t}\right) \xi, \xi\right\rangle\right|$. Then

$$
\|\operatorname{Re} \tilde{u}(x)\| \leq \sup \left|\left\langle u\left(e_{t}\right) \xi, \xi\right\rangle\right| \leq \sup _{t}\left\|u\left(e_{t}\right)\right\|,
$$

where the second last supremum is over $t$ and $\xi \in H,\|\xi\|=1$.

Corollary 5.20. Let $A$ be a unital real $J C^{*}$-algebra. If $u: A \rightarrow B(H)$ is selfadjoint and positive then $u$ is real bounded with $\|u\|_{r}=\left\|(\operatorname{Re} u)^{* *}(1)\right\|=\lim _{t}\left\|u\left(e_{t}\right)\right\|$. Here $\left(e_{t}\right)$ is a (increasing, if one wishes) cai for $A$.

Proof. Since $u$ is real positive we may appeal to Lemma 5.19. 
Corollary 5.21. Let $A, B$ be approximately unital real Jordan operator algebras, and let $T: A \rightarrow B$ be a contraction which is approximately unital (that is, takes some Jordan cai to a Jordan cai), or more generally for which $T^{* *}$ is unital. Then $T$ is real positive. If in addition $T$ is selfadjoint on $\Delta(A)$ then $T$ is systematically real positive.

If $\theta: A \rightarrow B$ is a contractive Jordan homomorphism then $\theta$ is systematically real positive.

Proof. By taking the second dual we may assume that $A, B$ are unital, and that $T(1)=1$. Then the first assertion follows from the lines before Example 5.10. The 'in addition' statement then follows from Theorem 5.6 or the paragraphs after that. The last assertion follows easily from the first after replacing $B$ with $\overline{\theta(A)}$, and noting that the restriction of $\theta$ to the real $\mathrm{JC}^{*}$-algebra $\Delta(A)$ is selfadjoint by Theorem 2.6. It also follows easily from Lemma 5.7

Remark 5.22. If $\theta: A \rightarrow B$ is a contractive (resp. isometric) Jordan homomorphism between unital or approximately unital real Jordan operator algebras then we are not certain if $\tilde{\theta}: A+A^{*} \rightarrow B+B^{*}$ is contractive (resp. isometric).

Theorem 5.23. Let $A$ and $B$ be approximately unital real Jordan operator algebras, and write $A^{1}$ for a real Jordan operator algebra unitization of $A$ with $A \neq A^{1}$. Let $C$ be a unital real Jordan operator algebra containing $B$ as a closed Jordan subalgebra.

(1) A real positive real contractive linear map $T: A \rightarrow B$ extends to a unital real positive linear map from $A^{1}$ to $C$, which is systematically real positive and real contractive if $T$ is also selfadjoint on $\Delta(A)$.

(2) A real completely positive completely contractive linear map $T: A \rightarrow B$ extends to a unital real completely positive completely contractive linear map from $A^{1}$ to $C$.

Proof. (1) We follow the proof of [10, Theorem 2.3], with a few tweaks. Clearly (2) follows from (1). Let $\tilde{T}: A^{1} \rightarrow C$ be the canonical unital extension of $T$ in (1), and write $e, f$ for the units of $A^{1}$ and $C$. So $\tilde{T}(a+s e)=T(a)+s f$ for $s \in \mathbb{R}, a \in A$. Suppose that $A$ is a Jordan subalgebra of some real $C^{*}$-algebra $D$. Since $e \notin A$ we may assume that $e=1_{D^{1}} \notin D$. Suppose that $\operatorname{Re}(x+\lambda e) \geq 0$ for $x \in A$ and scalar $\lambda$. We need to prove that $\operatorname{Re}(T(x)+\lambda f) \geq 0$. This is clear if $\operatorname{Re}$ $(\lambda)=0$, so suppose the contrary. Now Re $(\lambda)>0$ (by considering the character $\chi$ on $D^{1}$ that annihilates $D$; this is a state so that $\left.\operatorname{Re}(\chi(x)+\lambda)=\operatorname{Re}(\lambda) \geq 0\right)$. Since $\operatorname{Re}(x+\lambda e) \geq 0$ we have $-\frac{1}{\operatorname{Re}(\lambda)} \operatorname{Re}(x) \leq e$. Let

$$
x_{n}=-\frac{n-1}{n \operatorname{Re}(\lambda)} x, \quad y=\operatorname{Re}\left(x_{n}\right) \leq \frac{n-1}{n} e
$$

and $z=y_{+} \leq \frac{n-1}{n} e$. By Theorem 4.7 there exists a contraction $a \in A$ with $0 \leq$ $z \leq \operatorname{Re}(a) \leq e$. Now $\operatorname{Re}\left(a-x_{n}\right) \geq 0$, since $\operatorname{Re}\left(x_{n}\right)=y \leq y_{+}=z \leq \operatorname{Re}(a)$. Also $\|\operatorname{Re}(T(a))\| \leq 1$ since $a$ and $\operatorname{Re} T$ are contractions, and therefore $0 \leq \operatorname{Re}(T(a)) \leq f$. Also, $\operatorname{Re} T\left(a-x_{n}\right) \geq 0$, so that $\operatorname{Re}\left(T\left(x_{n}\right)\right) \leq \operatorname{Re}(T(a)) \leq f$. That is,

$$
-\frac{n-1}{n \operatorname{Re}(\lambda)} \operatorname{Re}(T(x)) \leq f .
$$

Letting $n \rightarrow \infty$ we have that $\operatorname{Re}(T(x)+\lambda f) \geq 0$ as desired. Hence $\tilde{T}$ is a unital real positive map, which is selfadjoint on $\Delta\left(A^{1}\right)$, and thus is real contractive by Lemma 5.19 
(2) We have that $T_{c}: A_{c} \rightarrow B_{c}$ is completely positive and completely contractive. Then apply [8, Proposition 2.2], and finally restrict to $A^{1}$ (since $\left.\left(A^{1}\right)_{c}=\left(A_{c}\right)^{1}\right)$.

Remark 5.24. Of course the extensions in the previous result are unique. As in the complex case [8, 10] one may apply these results to extend projections $P: A \rightarrow A$ to unital projections on $A^{1}$.

In the complex scalar case one gets a better result [10, Theorem 2.3]: A real positive contractive linear map $T: A \rightarrow B$ extends to a unital real positive contractive linear map on $A^{1}$. We do not know if this is true in the real case, even if $T$ is systematically real positive. If it were one would obtain the corollary that a bounded real linear map $T: A \rightarrow B$ between approximately unital Jordan operator algebras which is selfadjoint on $\Delta(A)$, is real positive and contractive if and only if $T\left(\mathfrak{F}_{A}\right) \subset \mathfrak{F}_{B}$. We do not know if this is true either, although certainly $T\left(\mathfrak{F}_{A}\right) \subset \mathfrak{F}_{B}$ implies that $T$ is real positive.

Acknowledgment. Several results here (in particularly, many in Sections 2-4) are from the May $2020 \mathrm{PhD}$ thesis of author W. Tepsan [44. Other complementary facts, alternative proofs, and additional theory may be found there, and there may also possibly be a forthcoming paper on that topic.

We thank M. Kalantar for several helpful discussions, which we mention in more detail in and around Theorem 2.11 (see also 1.1). We also thank Ángel Rodríguez Palacios for some comments.

In terms of future directions, the noncommutative topology of real Jordan operator algebras in the spirit of [9] looks like a fruitful topic that should be pursued elsewhere, as well as some other features of the real positive cone that have not been explored for the real case here or in 44 . There are no doubt some interesting 'operator space aspects' of the theory of real associative operator algebras that are worth developing. In addition, there are several open questions stated in this paper. Finally it would be very worthwhile to find many more interesting examples of real Jordan operator algebras, particularly if they might be important in quantum physics. It does not seem hard to find examples of real Jordan operator algebras beyond those already mentioned in this paper or 44. For example if one looks inside the upper triangular (real or complex) matrices $\mathcal{U}_{n}$ one quickly spots several new examples. E.g. the matrices in $\mathcal{U}_{2}(\mathbb{C})$ with $a_{22}=\overline{a_{11}}$ and $a_{12}$ real. Or certain matrices in $\mathcal{U}_{n}$ that are 'symmetric' with respect to the diagonal connecting $a_{n 1}$ to $a_{1 n}$.

\section{REFERENCES}

[1] Sh. A. Ayupov, A. A. Rakhimov, and Sh. M. Usmanov, Jordan, Real, and Lie Structures in Operator Algebras, vol. 418, MAIA, Kluwer Academic Publ., 1997.

[2] Sh. A. Ayupov, A. A. Rakhimov, and A. Kh. Abduvaitov, Real $W^{*}$-algebras with an abelian Hermitian part (Russian), Mat. Zametki 71 (2002), 473-476; translation in Math. Notes 71 (2002), 432-435.

[3] W. B. Arveson, Subalgebras of $C^{*}$-algebras, Acta Math. 123 (1969), 141-224.

[4] C. A. Bearden, D. P. Blecher and S. Sharma, On positivity and roots in operator algebras, Integral Equations Operator Theory 79 (2014), 555-566.

[5] D. P. Blecher, Generalization of $C^{*}$-algebra methods via real positivity for operator and Banach algebras, pages 35-66 in "Operator algebras and their applications: A tribute to Richard V. Kadison", (ed. by R.S. Doran and E. Park), vol. 671, Contemporary Mathematics, American Mathematical Society, Providence, R.I. 2016. 
[6] D. P. Blecher, Real positive maps and conditional expectations on operator algebras, p. 63102 in Positivity and its applications, (Eds. E. Kikianty, M. Mabula, M. Messerschmidt, J. H. van der Walt, and M. Wortel), Trends in Mathematics, Birkhäuser (2021).

[7] D. P. Blecher and C. Le Merdy, Operator algebras and their modules - an operator space approach, Oxford Univ. Press, Oxford (2004).

[8] D. P. Blecher and M. Neal, Completely contractive projections on operator algebras, Pacific J. Math. 283 (2016), 289-324.

[9] D. P. Blecher and M. Neal, Noncommutative topology and Jordan operator algebras, Mathematische Nachrichten 292 (2019), 481-510.

[10] D. P. Blecher and M. Neal, Contractive projections and real positive maps on operator algebras, Studia Math 256 (2021), 21-60.

[11] A. Böttcher and A. Pietsch, Orthogonal and skew-symmetric operators in real Hilbert space, Integral Equations Operator Theory 74 (2012), 497-511.

[12] D. P. Blecher and C. J. Read, Operator algebras with contractive approximate identities, J. Functional Analysis 261 (2011), 188-217.

[13] D. P. Blecher and C. J. Read, Operator algebras with contractive approximate identities II, J. Functional Analysis 264 (2013), 1049-1067.

[14] D. P. Blecher and C. J. Read, Order theory and interpolation in operator algebras, Studia Math. 225 (2014), 61-95.

[15] D. P. Blecher, Z-J. Ruan, and A. M. Sinclair, A characterization of operator algebras, J. Functional Analysis 89 (1990), 288-301.

[16] D. P. Blecher and Z. Wang, Jordan operator algebras: basic theory, Mathematische Nachrichten, 291 (2018), 1629-1654.

[17] D. P. Blecher and Z. Wang, Jordan operator algebras revisited, Mathematische Nachrichten 292 (2019), 2129-2136.

[18] D. P. Blecher and Z. Wang, Involutive operator algebras, Positivity 24 (2020), 13-53.

[19] M. Cabrera García and A. Rodríguez Palacios, Non-associative normed algebras. Vol. 1, The Vidav-Palmer and Gelfand-Naimark theorems. Encyclopedia of Mathematics and its Applications, 154. Cambridge University Press, Cambridge, 2014.

[20] C.-H. Chu, T. Dang, B. Russo, and B. Ventura, Surjective isometries of real $C^{*}$-algebras, Journal of the London Mathematical Society, 47(1993), 97-118.

[21] A. Connes, A factor not anti-isomorphic to itself, Bull. London Math. Soc., 7 (1975), 171174.

[22] E. G. Effros and Z-J. Ruan, Operator spaces, London Mathematical Society Monographs. New Series, 23. The Clarendon Press, Oxford University Press, New York, 2000.

[23] K. Goodearl, Notes on real and complex $C^{*}$-algebras, Shiva Mathematics Series vol. 5, Shiva Publishing Ltd., Nantwitch, 1982.

[24] M. Hamana, Injective envelopes of $C^{*}$-dynamical systems, Tohoku Math. J. 37 (1985), 463487.

[25] M. Hamana, Injective envelopes of dynamical systems, Toyama Math. J. 34 (2011), 23-86.

[26] H. Hanche-Olsen and E. Størmer, Jordan operator algebras, Pitman Publishing Inc., London, 1984.

[27] L. Ingelstam, Real Banach algebras, Arkiv för Matematik, 5 (1964), 239-270.

[28] J. M. Isidro, W. Kaup, and A. Rodríguez-Palacios, On real forms of J $B^{*}$-triples, Manuscripta Math. 86 (1995), 311-335.

[29] J. M. Isidro and A. Rodríguez-Palacios, On the definition of real $W^{*}$-algebras, Proc. Amer. Math. Soc. 124 (1996), 3407-3410.

[30] R. V. Kadison, Isometries of operator algebras, Ann. of Math. 54 (1951), 325-338.

[31] B. Li, Real operator algebras, World Scientific, River Edge, N.J., 2003.

[32] J. D. Maitland Wright, Jordan $C^{*}$-algebras, Michigan Math. J. 24 (1977), 291-302.

[33] M. S. Moslehian, G. A. Muñoz-Fernández, A. M. Peralta, and J. B. Seoane-Sepúlveda, Similarities and differences between real and complex Banach spaces: an overview and recent developments, preprint (2021), arXiv:2107.03740

[34] T. Palmer, Real $C^{*}$-algebras, Pacific J. Math, 35 (1970), 195-204.

[35] V. I. Paulsen, Completely bounded maps and operator algebras, Cambridge Studies in Advanced Math., 78, Cambridge University Press, Cambridge, 2002.

[36] G. K. Pedersen, $C^{*}$-algebras and their automorphism groups, Academic Press, London (1979). 
[37] G. Pisier, Introduction to operator space theory, London Math. Soc. Lecture Note Series, 294, Cambridge University Press, Cambridge, 2003.

[38] A. Rodríguez Palacios, Conditional expectations in complete normed complex algebras satisfying the von Neumann inequality, Revised preprint, 2021, to appear.

[39] J. Rosenberg, Structure and application of real $C^{*}$-algebras, Contemporary Mathematics, 671 (2016), 235-258.

[40] Z-J. Ruan, On real operator spaces, Acta Mathematica Sinica, 19 (2003), 485-496.

[41] Z-J. Ruan, Complexifications of real operator spaces, Illinois Journal of Mathematics, 47 (2003), 1047-1062.

[42] S. Sharma, Real operator algebras and real completely isometric theory, Positivity 18 (2014), 95-118.

[43] E. Størmer, Positive linear maps of operator algebras, Springer Monographs in Mathematics, Springer-Verlag (2013).

[44] W. Tepsan, Real operator spaces, real operator algebras, and real Jordan operator algebras, PhD thesis, University of Houston (May, 2020).

[45] D. Topping, Jordan algebras of self-adjoint operators, Bull. Amer. Math. Soc., 71 (1965), 160-164.

[46] Z. Wang, Theory of Jordan operator algebras and operator $*$-algebras, PhD thesis, University of Houston, 2019.

Department of Mathematics, University of Houston, Houston, TX 77204-3008, USA Email address, David P. Blecher: dpblecher@central.uh.edu

Chiang Mai University, 239 Nimmanhaemin Road, Suthep, Muang, Chiang Mai, ThaiLAND, 50200

Email address, Worawit Tepsan: worawit.tepsan@cmu.ac.th 